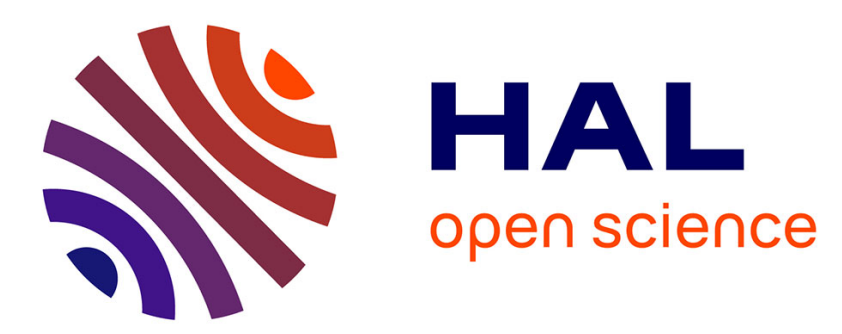

\title{
Shadow limit using Renormalization Group method and Center Manifold method
}

\author{
Anna Marciniak-Czochra, Andro Mikelic
}

\section{To cite this version:}

Anna Marciniak-Czochra, Andro Mikelic. Shadow limit using Renormalization Group method and Center Manifold method. Vietnam Journal of Mathematics, 2017, Special Issue of the Vietnam Journal of Mathematics dedicated to Willi Jaeger, 45, pp.103-125. hal-01280673

\section{HAL Id: hal-01280673 \\ https://hal.science/hal-01280673}

Submitted on 29 Feb 2016

HAL is a multi-disciplinary open access archive for the deposit and dissemination of scientific research documents, whether they are published or not. The documents may come from teaching and research institutions in France or abroad, or from public or private research centers.
L'archive ouverte pluridisciplinaire $\mathbf{H A L}$, est destinée au dépôt et à la diffusion de documents scientifiques de niveau recherche, publiés ou non, émanant des établissements d'enseignement et de recherche français ou étrangers, des laboratoires publics ou privés. 


\title{
Shadow limit using Renormalization Group method and Center Manifold method
}

\author{
Anna Marciniak-Czochra · Andro Mikelić
}

This contribution is dedicated to Professor Willi Jäger on his 75th birthday. Multiscale analysis and biological applications are two subjects offocus in Willi's research over his professional life.

\begin{abstract}
We study a shadow limit (the infinite diffusion coefficient-limit) of a system of ODEs coupled with a semilinear heat equation in a bounded domain with Neumann boundary conditions. In the literature, it was established formally that in the limit, the original semilinear heat equation reduces to an ODE involving the space averages of the solution to the semilinear heat equation and of the nonlinearity. It is coupled with the original system of ODEs for every space point $x$.We present derivation of the limit using the renormalization group (RG) and the center manifold approaches. The RG approach provides also further approximating expansion terms. The error estimate in the terms of the inverse of the diffusion coefficient is obtained for the finite time intervals. For the infinite times, the center manifolds for the starting problem and for its shadow limit approximation are compared and it is proved that their distance is of the order of the inverse of the diffusion coefficient.
\end{abstract}

Keywords Shadow limit - reaction-diffusion equations · model reduction · renormalization group $\cdot$ center manifold theorem

Accepted for publication in the Special Issue of the Vietnam Journal of Mathematics dedicated to Willi Jäger

Anna Marciniak-Czochra

Institute of Applied Mathematics, IWR and BIOQUANT, University of Heidelberg, Im Neuenheimer Feld 267, 69120 Heidelberg, Germany

Tel. : +496221544871

E-mail: anna.marciniak@iwr.uni-heidelberg.de

Andro Mikelić

Université de Lyon, CNRS UMR 5208, Université Lyon 1, Institut Camille Jordan, 43, blvd. du 11 novembre 1918, 69622 Villeurbanne Cedex, France

Tel. : +33426234548

Fax: +33956109885

E-mail: Andro.Mikelic@univ-lyon1.fr 
Mathematics Subject Classification (2000) 35B20 - 34E13 - 35B25 - 35B41 · $35 \mathrm{~K} 57$

\section{Introduction}

In the study of reaction-diffusion equations describing Turing-type pattern formation, it is necessary to consider very different diffusion coefficients. A number of such models was proposed in mathematical biology and chemistry, including the activatorinhibitor model of Gierer and Meinhardt [10], Gray-Scott model [8], Lengyel-Epstein model [18], and many others [24].

To study spatio-temporal evolution of solutions of such models, it is worthy to consider a reduced version of the model by letting the large diffusion coefficient tend infinity. The resulting system is useful only if it is a good approximation of the original dynamics and preserves the phenomenon of pattern formation. Such model reduction has been recently proposed also in analysis of reaction-diffusion-ode models with a single diffusion [20]. Reaction-diffusion-ode models, called also receptorbased models, arise in description of interactions between intracellular or cell dynamics regulated by a diffusive signaling factor. They have already been employed in various biological contexts, see eg. $[14,16,22,23]$. In this paper we focus on a rigorous proof of a large diffusion limit for such models.

A representative example is an ODE system, coupled with a semilinear parabolic equation with a large diffusion coefficient. Its ratio to the other coefficients is equal to the inverse of a small parameter $\varepsilon>0$. In the analysis of the model, we follow the approach established for problems having two characteristic times. We assume that $\Omega$ is a given open bounded set with a smooth boundary and focus on the Cauchy problem

$$
\begin{aligned}
\frac{\partial \mathbf{u}_{\varepsilon}}{\partial t} & =\mathbf{f}\left(\mathbf{u}_{\varepsilon}, v_{\varepsilon}\right), \quad \text { in } \quad(0, T) \times \Omega ; \quad \mathbf{u}_{\varepsilon}(0)=\mathbf{u}^{0}(x), x \in \Omega, \\
\frac{\partial v_{\varepsilon}}{\partial t} & =\frac{1}{\varepsilon} \Delta v_{\varepsilon}+\Phi\left(\mathbf{u}_{\varepsilon}, v_{\varepsilon}\right), \quad \text { in } \quad(0, T) \times \Omega, \quad v_{\varepsilon}(0)=v^{0}(x), x \in \Omega \\
\frac{\partial v_{\varepsilon}}{\partial v} & =0 \quad \text { in } \quad(0, T) \times \partial \Omega .
\end{aligned}
$$

Asymptotic analysis of problem (1)-(3) with $\varepsilon \rightarrow 0$ has attracted a considerable interest in the literature in the case where the first equation is a quasilinear parabolic equation, starting from the papers of Keener [15] and Hale [11].

In our case, the shadow limit reduction of equations (1)-(3) yields the following system of integro-differential equations

$$
\begin{aligned}
& \frac{\partial \mathbf{u}}{\partial t}=\mathbf{f}(\mathbf{u}, v) \\
& \frac{d v}{d t}=\frac{1}{|\Omega|} \int_{\Omega} \Phi(\mathbf{u}(x, t), v(t)) d x .
\end{aligned}
$$

For a finite time intervals, convergence of solutions of the $\varepsilon$-problem (1)-(3) to the solution of the shadow problem (4)- (5) was shown by Marciniak-Czochra and collaborators in ref. [20]. An approach using semigroup convergence has been recently 
established by Bobrowski in ref. [1]. However, its application to system (1)-(3) required some properties of the solutions which are not satisfied in general.

In this article we present a detailed study of the limit process by comparing solutions of the two systems (1)-(3) and (4)-(5) and proving an error estimate in terms of $\varepsilon$. The employed methods are the renormalization group technique (RG) and the center manifold theorem.

The paper is organized as follows. We formally derive the renormalization group (RG) equation in Section 2. It yields the shadow limit equation and, also, allows to determine the next order correction term. Next, in Section 3 we prove the approximation for finite time intervals. The results are given in Theorem 3 , which is proven in two steps. First we construct appropriate cut-offs and a barrier function and prove that the difference of solutions is of the order $O(\varepsilon)$ in $L^{\infty}(\Omega \times(0, T)$. Then, using energy estimates, the perturbation from the mean for the semilinear heat equation (2) is proven to be of the order $O\left(\varepsilon^{3 / 2}\right)$ in $L^{2}\left(0, T ; H^{1}(\Omega)\right)$. In Section 4 we determine the center manifold around a critical point for both systems (1)-(3) and (4)-(5). The main result is obtained in Theorem 4 through a comparison between the constructed center manifolds. We give their construction and prove that (i) their central spectra coincide, (ii) the "master" equation is the same and (iii) the reduction function for the perturbation part from the mean for the semilinear heat equation (2) is of the order $O(\varepsilon)$ in the sup-norm.

\section{RG approach to the shadow limit}

The RG method originates from theoretical physics. It was introduced for singular perturbation problems by Chen, Goldenfeld and Oono in references [3,4], where the method was formally applied to several examples. One advantage of the RG method is that it provides an algorithm for derivation of reduced models. Its first step is a straightforward perturbation expansion. The expansion usually involves secular terms that exhibit unbounded growth in time, which can be however removed by the appropriate reparametrization provided by the $\mathrm{RG}$ equations. The procedure leads to correct asymptotic expansions. The RG method allows to identify all multiple scales present in the problem and provides the result based on a systematic procedure. The involved computations may be tedious, but they are straightforward.

A mathematically rigorous theory of the RG method was developed for systems of ordinary differential equations of the form

$$
\frac{d \mathbf{x}}{d t}=F \mathbf{x}+\varepsilon \mathbf{h}(\mathbf{x}, t)
$$

valid for long time intervals. Here $F$ is a matrix with purely imaginary eigenvalues. In the naive expansion approach secular terms appear and asymptotic expansions are not valid for the time intervals of the length $T=O\left(\frac{1}{\varepsilon}\right)$. It was shown in references [5], [6] and [9] that the RG method provides a good approximation also for long times. Furthermore they pointed out that the RG method unifies the multiple time scale expansion techniques, the center manifold theory, the geometric singular perturbation 
and other perturbation methods. The RG method has been also applied to some partial differential equations, in particular, to the geostrophic flows, see ref. [29], [25], [26]. In a recent article [7] Chiba considered approximation of the perturbed higher order nonlinear parabolic PDEs by simpler amplitude parabolic PDEs. The shadow limit approximation does not enter into that class of problems. Nevertheless, in this section we will give a formal derivation of the shadow limit system (4)-(5) from (1)(3) using the RG approach. Once the time variable is rescaled, we see immediately the analogy with the above quoted works. We construct the RG approximation, which is a simple case of the RG transform $\alpha_{t}$ from Chiba's articles. It contains the solution to the shadow limit problem (4)-(5) and the "coordinate transformation " $\varepsilon w^{\Phi} \cdot w^{\Phi}$ controls the "slave" modes in the nonlinearity $\Phi$.

In this paper, we consider the Cauchy' problem (1)-(3). The nonlinearities $\mathbf{f}$ and $\Phi$ are defined on $\mathbb{R}^{m+1}, m \geq 1$, and take values in $\mathbb{R}^{m}$ and $\mathbb{R}^{1}$, respectively. It is assumed that they are $C^{2}$ with bounded derivatives and that problem (1)-(3) has a unique globally defined smooth solution.

In order to apply the renormalization group (RG) approach, we change time scale by setting $\tau=t / \varepsilon$. System (1)-(3) becomes

$$
\begin{aligned}
& \frac{\partial \mathbf{u}_{\varepsilon}}{\partial \tau}=\varepsilon \mathbf{f}\left(\mathbf{u}_{\varepsilon}, v_{\varepsilon}\right) \quad \text { and } \quad \frac{\partial v_{\varepsilon}}{\partial \tau}-\Delta v_{\varepsilon}=\varepsilon \Phi\left(\mathbf{u}_{\varepsilon}, v_{\varepsilon}\right), \quad \text { in } \quad \Omega \times(0, T) \\
& \frac{\partial v_{\varepsilon}}{\partial v}=0 \quad \text { on } \quad(0, T) \times \partial \Omega, \quad \mathbf{u}_{\varepsilon}(0)=\mathbf{u}^{0} \quad \text { and } \quad v_{\varepsilon}(0)=v^{0} \quad \text { in } \quad \Omega .
\end{aligned}
$$

\subsection{Prerequisites}

Before presenting the RG calculations we recall two elementary results from the parabolic theory and a simple lemma about ODEs with an exponentially decaying right-hand side.

1. We consider the spectral problem:

Find $w \in H^{1}(\Omega)$, which is not identically equal to zero, and $\lambda \in \mathbb{R}$ such that

$$
-\Delta w=\lambda w \quad \text { in } \quad \Omega ; \quad \frac{\partial w}{\partial v}=0 \quad \text { on } \quad \partial \Omega .
$$

It admits a countable set of eigenvalues and eigenfunctions $\left\{\lambda_{j}, w_{j}\right\}$. The principal eigenvalue $\lambda_{0}=0$ and $w_{0}=1 / \sqrt{|\Omega|}$. $\lambda_{j}$ tends to infinity as $j \rightarrow \infty$. The eigenfunctions $\left\{w_{j}\right\}$ form an orthonormal basis for $L^{2}(\Omega)$ and an orthogonal basis for $H^{1}(\Omega)$.

2. Let $U_{0} \in L^{2}(\Omega)$ and $F \in L^{2}(\Omega \times(0, T))$. Then, the initial/boundary-value problem

$$
\begin{aligned}
& \frac{\partial U}{\partial t}-\Delta U=F(x, t) \quad \text { in } \quad \Omega \times(0, T), \\
& \text { (9) parab1 } \\
& \frac{\partial U}{\partial v}=0 \quad \text { on } \quad \partial \Omega \times(0, T),\left.\quad U\right|_{t=0}=U_{0} \quad \text { in } \quad \Omega, \\
& \text { (10) parab2 }
\end{aligned}
$$


has a unique variational solution $U \in L^{2}\left(0, T ; H^{1}(\Omega)\right) \cap C\left([0, T] ; L^{2}(\Omega)\right)$, given by the separation of variables formula

$$
\begin{aligned}
U(x, t) & =\left\langle U_{0}+\int_{0}^{t} F(\cdot, s) d s\right\rangle_{\Omega}+\sum_{j=1}^{\infty}\left\{\left(U_{0}, w_{j}\right)_{L^{2}(\Omega)} e^{-\lambda_{j} t}\right. \\
& \left.+\int_{0}^{t}\left(F(\cdot, s), w_{j}\right)_{L^{2}(\Omega)} e^{-\lambda_{j}(t-s)} d s\right\} w_{j}(x),
\end{aligned}
$$

where the arithmetic mean is

$$
\langle z\rangle_{\Omega}=\frac{1}{|\Omega|} \int_{\Omega} z(x) d x, \quad z \in L^{1}(\Omega) .
$$

3. Lemma 1 Let $g: \mathbb{R} \times[0,+\infty) \rightarrow \mathbb{R}, g=g(y, t)$ be a continuous function that is Lipschitz in $y$ and satisfies the inequality

$$
|g(y, t)| \leq C e^{-\gamma t}, \quad \text { for some } \gamma>0 \quad \text { and all } t \in[0,+\infty) .
$$

Then, the solution y for the Cauchy problem

$$
\frac{d y}{d t}=g(y, t), \quad y(0)=y_{0}
$$

is a globally defined function such that

$$
\|y\|_{L^{\infty}\left(\mathbb{R}_{+}\right)} \leq C \quad \text { and } \quad \sup _{t \in \mathbb{R}_{+}}\left|\int_{0}^{t} y(s) d s-t y(t)\right| \leq C .
$$

\subsection{Application of RG method}

We now proceed by usual RG method steps.

1. We assume that the problem can be solved as a regular perturbation problem and calculate the straightforward expansion

$$
\begin{aligned}
& \mathbf{u}_{\varepsilon}(x, \tau)=\mathbf{u}_{0}(x, \tau)+\varepsilon \mathbf{u}_{1}(x, \tau)+\varepsilon^{2} \mathbf{u}_{2}(x, \tau)+\ldots, \\
& v_{\varepsilon}(x, \tau)=v_{0}(x, \tau)+\varepsilon v_{1}(x, \tau)+\varepsilon^{2} v_{2}(x, \tau) \ldots
\end{aligned}
$$

It yields

$$
\begin{gathered}
\frac{\partial}{\partial \tau}\left(\mathbf{u}_{0}+\varepsilon \mathbf{u}_{1}+\varepsilon^{2} \mathbf{u}_{2}+\ldots\right)=\varepsilon \mathbf{f}\left(\mathbf{u}_{0}, v_{0}\right)+ \\
\varepsilon^{2}\left(\nabla_{u} \mathbf{f}\left(\mathbf{u}_{0}, v_{0}\right) \mathbf{u}_{1}(\tau)+\partial_{v} \mathbf{f}\left(\mathbf{u}_{0}, v_{0}\right) v_{1}(\tau)\right)+\ldots, \\
\left(\frac{\partial}{\partial \tau}-\Delta_{x}\right)\left(v_{0}+\varepsilon v_{1}+\varepsilon^{2} v_{2} \ldots\right)=\varepsilon \Phi\left(\mathbf{u}_{0}, v_{0}\right)+ \\
\varepsilon^{2}\left\{\nabla_{u} \Phi\left(\mathbf{u}_{0}, v_{0}\right) \cdot \mathbf{u}_{1}(\tau)+\frac{\partial \Phi}{\partial v}\left(\mathbf{u}_{0}, v_{0}\right) v_{1}(\tau)\right\}+\ldots
\end{gathered}
$$


Comparing the terms of the order zero we obtain

$$
\left(\frac{\partial}{\partial \tau}-\Delta_{x}\right) v_{0}=0 \quad \text { and } \quad \frac{\partial}{\partial \tau} \mathbf{u}_{0}=0
$$

Equations (15) yield

$$
\mathbf{u}_{0}(x, \tau)=\mathbf{A}(x) \quad \text { and } \quad v_{0}(x, \tau)=B+\sum_{j=1}^{+\infty} b_{j} w_{j}(x) e^{-\lambda_{j} \tau}
$$

where $B$ is a constant.

On the order $O(\varepsilon)$, we obtain

$$
\begin{gathered}
\left(\frac{\partial}{\partial \tau}-\Delta_{x}\right) v_{1}=\Phi\left(\mathbf{A}, v_{0}\right), \quad \text { in } \quad \Omega \times(0, T), \\
\frac{\partial}{\partial \tau} \mathbf{u}_{1}=\mathbf{f}\left(\mathbf{A}, v_{0}\right), \quad \text { in } \quad \Omega \times(0, T) .
\end{gathered}
$$

We note that $\mathbf{f}\left(\mathbf{A}, v_{0}\right)=\mathbf{f}\left(\mathbf{A}, v_{0}\right)-\mathbf{f}(\mathbf{A}, B)+\mathbf{f}(\mathbf{A}, B)$, with $\left|\mathbf{f}\left(\mathbf{A}, v_{0}(\tau)\right)-\mathbf{f}(\mathbf{A}, B)\right| \leq$ $C \exp \left\{-\lambda_{1} \tau\right\}$. Hence, equation (18) leads to

$$
\mathbf{u}_{1}(x, \tau)=\tau \mathbf{f}(\mathbf{A}, B)+\mathbf{C}_{1 u}(x, \tau),
$$

where $\mathbf{C}_{1 u}$ is a solution to the Cauchy problem (13) with $g_{j}=f_{j}\left(\mathbf{A}, v^{0}\right)-f_{j}(\mathbf{A}, B)$. By Lemma 1, the function $\mathbf{C}_{1 u}$ is uniformly bounded with respect to $\tau$.

We use equation (17) to calculate $v_{1}$. Once again, we decompose the right-hand side as $\Phi\left(\mathbf{A}, v_{0}\right)=\Phi(\mathbf{A}, B)+\Phi\left(\mathbf{A}, v_{0}\right)-\Phi(\mathbf{A}, B)$. Using formula (11), with the right-hand side $\Phi\left(\mathbf{A}, v_{0}\right)-\Phi(\mathbf{A}, B)$ exponentially decreasing in $\tau$, yields a solution that is uniformly bounded in $\tau \in \mathbb{R}_{+}$. The right-hand side $\Phi(\mathbf{A}, B)$ is bounded with respect to $\tau$ and it contributes as an affine term in $\tau$ :

$$
v_{1}(x, \tau)=\tau\langle\Phi(\mathbf{A}, B)\rangle_{\Omega}+\sum_{j=1}^{+\infty}\left(\Phi(\mathbf{A}, B), w_{j}\right)_{L^{2}(\Omega)} \frac{w_{j}(x)}{\lambda_{j}}+C_{1 v},
$$

where $C_{1 v}$ is a solution of the Cauchy problem (13) with $g|\Omega|=\int_{\Omega}\left(\Phi\left(\mathbf{A}, v^{0}\right)-\right.$ $\Phi(\mathbf{A}, B)) d x$.

By Lemma 1 , the function $C_{1 v}$ is uniformly bounded with respect to $\tau$.

Comparing the terms of the order $O\left(\varepsilon^{2}\right)$ for $\mathbf{u}_{2}$, we obtain

$$
\begin{gathered}
\frac{\partial}{\partial \tau} \mathbf{u}_{2}(x, \tau)=\nabla_{u} \mathbf{f}\left(\mathbf{u}_{0}, v_{0}\right) \mathbf{u}_{1}(x, \tau)+\partial_{v} \mathbf{f}\left(\mathbf{u}_{0}, v_{0}\right) v_{1}(x, \tau)= \\
\tau\left(\nabla_{u} \mathbf{f}(\mathbf{A}, B) \mathbf{f}(\mathbf{A}, B)+\partial_{v} \mathbf{f}(\mathbf{A}, B)\langle\Phi(\mathbf{A}, B)\rangle_{\Omega}\right)+\nabla_{u} \mathbf{f}(\mathbf{A}, B) \mathbf{C}_{1 u}(x, \tau)+ \\
\partial_{v} \mathbf{f}(\mathbf{A}, B)\left(C_{1 v}(x, \tau)+\sum_{j=1}^{+\infty}\left(\Phi(\mathbf{A}, B), w_{j}\right)_{L^{2}(\Omega)} \frac{w_{j}(x)}{\lambda_{j}}\right)+O\left((1+\tau) e^{-\lambda_{1} \tau}\right),
\end{gathered}
$$


and consequently

$$
\begin{gathered}
\mathbf{u}_{2}(x, \tau)=\frac{\tau^{2}}{2}\left(\nabla_{u} \mathbf{f}(\mathbf{A}, B) \mathbf{f}(\mathbf{A}, B)+\partial_{v} \mathbf{f}(\mathbf{A}, B)\langle\Phi(\mathbf{A}, B)\rangle_{\Omega}\right)+ \\
\tau\left(\nabla_{u} \mathbf{f}(\mathbf{A}, B) \mathbf{C}_{1 u}(x, \tau)+\partial_{v} \mathbf{f}(\mathbf{A}, B)\left(C_{1 v}(x, \tau)+\sum_{j=1}^{+\infty}\left(\Phi(\mathbf{A}, B), w_{j}\right)_{L^{2}(\Omega)} \frac{w_{j}(x)}{\lambda_{j}}\right)\right) \\
+\mathbf{C}_{2 u}(x, \tau) .
\end{gathered}
$$

By estimates (14), the function $\mathbf{C}_{2 u}(x, \tau)$ is uniformly bounded in $\tau$.

Next, comparing the terms of the order $O\left(\varepsilon^{2}\right)$ for $\mathbf{v}_{2}$, we obtain

$$
\begin{aligned}
& \left(\frac{\partial}{\partial \tau}-\Delta_{x}\right) v_{2}(x, \tau)=\nabla_{u} \Phi\left(\mathbf{A}, v_{0}\right) \cdot \mathbf{u}_{1}+\partial_{v} \Phi\left(\mathbf{A}, v_{0}\right) v_{1}=\tau\left(\nabla_{u} \Phi(\mathbf{A}, B) \cdot \mathbf{f}(\mathbf{A}, B)\right. \\
& \left.\quad+\partial_{v} \Phi(\mathbf{A}, B)\langle\Phi(\mathbf{A}, B)\rangle_{\Omega}\right)+\nabla_{u} \Phi(\mathbf{A}, B) \cdot \mathbf{C}_{1 u}+\partial_{v} \Phi(\mathbf{A}, B)\left(C_{1 v}(x, \tau)\right. \\
& \left.\quad+\sum_{j=1}^{+\infty}\left(\Phi(\mathbf{A}, B), w_{j}\right)_{L^{2}(\Omega)} \frac{w_{j}(x)}{\lambda_{j}}\right)+O\left((1+\tau) e^{-\lambda_{1} \tau}\right) \quad \text { in } \quad \Omega \times(0, T),
\end{aligned}
$$

together with boundary and initial conditions (10). The separation of variables formula (11) and Lemma 1 yield

$$
\begin{gathered}
v_{2}(x, \tau)=\frac{\tau^{2}}{2}\left(\left\langle\nabla_{u} \Phi(\mathbf{A}, B) \cdot \mathbf{f}(\mathbf{A}, B)\right\rangle_{\Omega}+\left\langle\partial_{v} \Phi(\mathbf{A}, B)\right\rangle_{\Omega}\langle\Phi(\mathbf{A}, B)\rangle_{\Omega}\right)+ \\
\tau\left(\left\langle\nabla_{u} \Phi(\mathbf{A}, B) \cdot \mathbf{C}_{1 u}\right\rangle_{\Omega}+\left\langle\partial_{v} \Phi(\mathbf{A}, B)\left(C_{1 v}(x, \tau)+\sum_{j=1}^{+\infty}\left(\Phi(\mathbf{A}, B), w_{j}\right)_{L^{2}(\Omega)} \frac{w_{j}(x)}{\lambda_{j}}\right)\right\rangle_{\Omega}\right) \\
+\tau \sum_{j=1}^{+\infty} \frac{w_{j}(x)}{\lambda_{j}}\left(\left(\nabla_{u} \Phi(\mathbf{A}, B) \cdot \mathbf{f}(\mathbf{A}, B), w_{j}\right)_{L^{2}(\Omega)}+\left(\partial_{v} \Phi(\mathbf{A}, B), w_{j}\right)_{L^{2}(\Omega)}\langle\Phi(\mathbf{A}, B)\rangle_{\Omega}\right) \\
+C_{2 v}(x, \tau) .
\end{gathered}
$$

By estimates (14), the function $C_{2 v}(x, \tau)$ is uniformly bounded in $\tau$.

The approximation takes the form

$$
\begin{aligned}
\mathbf{u}_{\varepsilon}(x, \tau) & =\mathbf{u}_{0}(x, \tau)+\varepsilon \mathbf{u}_{1}(x, \tau)+\varepsilon^{2} \mathbf{u}_{2}(x, \tau)+O\left(\varepsilon^{3}\right), \\
v_{\varepsilon}(x, \tau) & =v_{0}(x, \tau)+\varepsilon v_{1}(x, \tau)+\varepsilon^{2} v_{2}(x, \tau)+O\left(\varepsilon^{3}\right) .
\end{aligned}
$$

Since solutions $\mathbf{u}_{1}, v_{1}, \mathbf{u}_{2}$ and $v_{2}$ involve terms with polynomials in $\tau$ which yield secular terms in the expansion (23), the approximation is valid only for time intervals with length of the order $O(1)$. In order to have an approximation valid for longer time intervals, it is necessary to eliminate the secular terms.

2. The idea of the renormalization is to introduce an arbitrary time $\mu$, split $\tau$ as $\tau-$ $\mu+\mu$ and absorb the terms containing $\mu$ into the renormalized counterparts $\mathbf{A}(\mu)$ and $B(\mu)$ of $\mathbf{A}$ and $B$, respectively. We introduce two renormalization constants

$$
\begin{aligned}
& \mathbf{Z}^{1}=1+\mathbf{a}_{1} \varepsilon+\mathbf{a}_{2} \varepsilon^{2}, \\
& Z_{2}=1+b_{1} \varepsilon+b_{2} \varepsilon^{2} .
\end{aligned}
$$


We renormalize the coefficients $\mathbf{A}$ and $B$ using the following expansions

$$
\begin{aligned}
A_{k} & =\left(1+a_{1 k} \varepsilon+a_{2 k} \varepsilon^{2}\right) A_{k}(\mu), \quad k=1, \ldots, m, \\
B & =\left(1+b_{1} \varepsilon+b_{2} \varepsilon^{2}\right) B(\mu) .
\end{aligned}
$$

The coefficients $\mathbf{a}_{2}, \mathbf{a}_{1}, b_{2}$ and $b_{1}$ can be chosen to eliminate the terms containing the secular terms $\mu \varepsilon, \mu \varepsilon^{2}$ and $\mu^{2} \varepsilon^{2}$. Consequently, only the terms in $\tau-\mu$ remain in the approximation.

Inserting formulas (24) into the approximation (22)-(23), we obtain

$$
\begin{aligned}
& u^{k}(\tau, \mu)=u_{0 k}(\tau)+\varepsilon u_{1 k}(\tau)+\varepsilon^{2} u_{2 k}(\tau)=\left(1+a_{1 k} \varepsilon+a_{2 k} \varepsilon^{2}\right) A_{k}(\mu)+ \\
& \varepsilon(\tau-\mu+\mu)\left(f_{k}(\mathbf{A}, B)+\varepsilon \sum_{j=1}^{m} \partial_{u_{j}} f_{k}(\mathbf{A}, B) a_{1 j} A_{j}(\mu)+\varepsilon B b_{1} \partial_{v} f_{k}(\mathbf{A}, B)\right)+ \\
& \varepsilon C_{1 u, k}+\frac{\varepsilon^{2}}{2}\left(\tau^{2}-\mu^{2}+\mu^{2}\right)\left(\nabla_{u} \mathbf{f}(\mathbf{A}, B) \mathbf{f}(\mathbf{A}, B)+\partial_{v} \mathbf{f}(\mathbf{A}, B)\langle\Phi(\mathbf{A}, B)\rangle_{\Omega}\right)_{k}+ \\
& \varepsilon^{2}(\tau-\mu+\mu)\left(\left(\nabla_{u} \mathbf{f}(\mathbf{A}, B) \mathbf{C}_{1 u}(x, \tau)\right)_{k}+\partial_{v} f_{k}(\mathbf{A}, B)\left(C_{1 v}(x, \tau)+\right.\right. \\
& \left.\left.\sum_{j=1}^{+\infty}\left(\Phi(\mathbf{A}, B), w_{j}\right)_{L^{2}(\Omega)} \frac{w_{j}(x)}{\lambda_{j}}\right)\right)+\varepsilon^{2} C_{2 u, k}(x, \tau), \quad k=1, \ldots, m . \\
& v(\tau, \mu)=v_{0}(\tau)+\varepsilon v_{1}(\tau)+\varepsilon^{2}(\tau)=B(\mu)\left(1+b_{1} \varepsilon+b_{2} \varepsilon^{2}\right)+\varepsilon(\tau-\mu \\
& +\mu)\left(\langle\Phi(\mathbf{A}, B)\rangle_{\Omega}+\varepsilon \sum_{j=1}^{m}\left\langle\partial_{u_{j}} \Phi(\mathbf{A}, B) a_{1 j} A_{j}(\mu)\right\rangle_{\Omega}+\varepsilon\left\langle B b_{1} \partial_{v} \Phi(\mathbf{A}, B)\right\rangle_{\Omega}\right) \\
& +\varepsilon C_{1 v}+\varepsilon \sum_{j=1}^{+\infty}\left(\Phi\left(\mathbf{A}\left(1+\varepsilon \mathbf{a}^{1}\right), B\left(1+\varepsilon b_{1}\right)\right), w_{j}\right)_{L^{2}(\Omega)} \frac{w_{j}(x)}{\lambda_{j}}+ \\
& \frac{\varepsilon^{2}}{2}\left(\tau^{2}-\mu^{2}+\mu^{2}\right)\left(\left\langle\nabla_{u} \Phi(\mathbf{A}, B) \cdot \mathbf{f}(\mathbf{A}, B)\right\rangle_{\Omega}+\left\langle\partial_{v} \Phi(\mathbf{A}, B)\right\rangle_{\Omega}\langle\Phi(\mathbf{A}, B)\rangle_{\Omega}\right)+ \\
& \varepsilon^{2}(\tau-\mu+\mu)\left(\left\langle\nabla_{u} \Phi(\mathbf{A}, B) \cdot \mathbf{C}_{1 u}\right\rangle_{\Omega}+\left\langle\partial _ { \nu } \Phi ( \mathbf { A } , B ) \left( C_{1 v}(x, \tau)+\right.\right.\right. \\
& \left.\left.\sum_{j=1}^{+\infty}\left(\Phi(\mathbf{A}, B), w_{j}\right)_{L^{2}(\Omega)} \frac{w_{j}(x)}{\lambda_{j}}\right)\right\rangle_{\Omega}+\sum_{j=1}^{+\infty} \frac{w_{j}(x)}{\lambda_{j}}\left(\left(\nabla_{u} \Phi(\mathbf{A}, B) \cdot \mathbf{f}(\mathbf{A}, B), w_{j}\right)_{L^{2}(\Omega)}+\right. \\
& \left.\left.\left(\partial_{v} \Phi(\mathbf{A}, B), w_{j}\right)_{L^{2}(\Omega)}\langle\Phi(\mathbf{A}, B)\rangle_{\Omega}\right)\right)+\varepsilon^{2} C_{2 v}(x, \tau)+O\left(e^{-\lambda \tau}\right)+O\left(\varepsilon^{3}\right) .
\end{aligned}
$$

Next, we choose the renormalization constants $a_{1 k}$ and $b_{1}$ in such way that the secular terms in $\mu$ of the order $O(\varepsilon)$ are eliminated. Consequently, we obtain

$$
a_{1 k}(\mu) A_{k}(\mu)+\mu f_{k}(\mathbf{A}, B)+C_{1 u, k}=0,
$$

implying

$$
a_{1 k}=-\frac{\mu}{A_{k}} f_{k}(\mathbf{A}, B)-\frac{C_{1 u, k}}{A_{k}}, \quad k=1, \ldots, m .
$$


Analogously, for $b_{1}$ it holds

$$
b_{1}=-\frac{\mu}{B}\langle\Phi(\mathbf{A}, B)\rangle_{\Omega}-\frac{C_{1 v}}{B}-\frac{1}{B} \sum_{j=1}^{+\infty}\left(\Phi(\mathbf{A}, B), w_{j}\right)_{L^{2}(\Omega)} \frac{w_{j}(x)}{\lambda_{j}}
$$

Now we insert formulas (27) and (28) into (25)-(26). The terms of the order $O\left(\varepsilon^{2}\right)$, containing only $\mu$ and its powers (the secular terms), are to be eliminated and only terms containing $\tau-\mu$ and $\tau^{2}-\mu^{2}$ should remain. We achieve this goal by choosing appropriate $\left\{a_{2 k}\right\}_{k=1, \ldots, m}$ and $b_{2}$, given explicitly by comparing the corresponding terms of the order $O\left(\varepsilon^{2}\right)$. The resulting expressions for $\left\{u^{k}(\tau, \mu)\right\}_{k=1, \ldots, m}$ are

$$
\begin{gathered}
u^{k}(\tau, \mu)=A_{k}(\mu)+\varepsilon(\tau-\mu)\left(f_{k}(\mathbf{A}, B)-\varepsilon \sum_{j=1}^{m} \partial_{u_{j}} f_{k}(\mathbf{A}, B)\left(\mu f_{j}(\mathbf{A}, B)+C_{1 u, j}\right)-\right. \\
\left.\varepsilon \partial_{v} f_{k}(\mathbf{A}, B)\left(\mu\langle\Phi(\mathbf{A}, B)\rangle_{\Omega}+C_{1 v}(x, \tau)+\sum_{j=1}^{+\infty}\left(\Phi(\mathbf{A}, B), w_{j}\right)_{L^{2}(\Omega)} \frac{w_{j}(x)}{\lambda_{j}}\right)\right)+ \\
\frac{\varepsilon^{2}}{2}\left(\tau^{2}-\mu^{2}\right)\left(\nabla_{u} \mathbf{f}(\mathbf{A}, B) \mathbf{f}(\mathbf{A}, B)+\partial_{v} \mathbf{f}(\mathbf{A}, B)\langle\Phi(\mathbf{A}, B)\rangle_{\Omega}\right)_{k}+ \\
\varepsilon^{2}(\tau-\mu)\left(\left(\nabla_{u} \mathbf{f}(\mathbf{A}, B) \mathbf{C}_{1 u}(x, \tau)\right)_{k}+\partial_{v} f_{k}(\mathbf{A}, B)\left(C_{1 v}(x, \tau)+\right.\right. \\
\left.\left.\sum_{j=1}^{+\infty}\left(\Phi(\mathbf{A}, B), w_{j}\right)_{L^{2}(\Omega)} \frac{w_{j}(x)}{\lambda_{j}}\right)\right), \quad k=1, \ldots, m .
\end{gathered}
$$

and for $v(\tau, \mu)$

$$
\begin{gathered}
v(\tau, \mu)=B(\mu)+\varepsilon(\tau-\mu)\left(\langle\Phi(\mathbf{A}, B)\rangle_{\Omega}-\varepsilon \sum_{j=1}^{m}\left\langle\partial_{u_{j}} \Phi(\mathbf{A}, B)\left(\mu f_{j}(\mathbf{A}, B)+C_{1 u, j}\right)\right\rangle_{\Omega}\right. \\
\left.-\varepsilon\left\langle\partial_{v} \Phi(\mathbf{A}, B)\left(\mu\langle\Phi(\mathbf{A}, B)\rangle_{\Omega}+C_{1 v}(x, \tau)+\sum_{j=1}^{+\infty}\left(\Phi(\mathbf{A}, B), w_{j}\right)_{L^{2}(\Omega)} \frac{w_{j}(x)}{\lambda_{j}}\right)\right\rangle_{\Omega}\right)+ \\
\frac{\varepsilon^{2}}{2}\left(\tau^{2}-\mu^{2}\right)\left(\left\langle\nabla_{u} \Phi(\mathbf{A}, B) \cdot \mathbf{f}(\mathbf{A}, B)\right\rangle_{\Omega}+\left\langle\partial_{v} \Phi(\mathbf{A}, B)\right\rangle_{\Omega}\langle\Phi(\mathbf{A}, B)\rangle_{\Omega}\right)+ \\
\varepsilon^{2}(\tau-\mu)\left(\left\langle\nabla_{u} \Phi(\mathbf{A}, B) \cdot \mathbf{C}_{1 u}\right\rangle_{\Omega}+\left\langle\partial _ { v } \Phi ( \mathbf { A } , B ) \left( C_{1 v}(x, \tau)+\right.\right.\right. \\
\left.\left.\sum_{j=1}^{+\infty}\left(\Phi(\mathbf{A}, B), w_{j}\right)_{L^{2}(\Omega)} \frac{w_{j}(x)}{\lambda_{j}}\right)\right\rangle_{\Omega}+\sum_{j=1}^{+\infty} \frac{w_{j}(x)}{\lambda_{j}}\left(\left(\nabla_{u} \Phi(\mathbf{A}, B) \cdot \mathbf{f}(\mathbf{A}, B), w_{j}\right)_{L^{2}(\Omega)}+\right. \\
\left.\left.\left(\partial_{v} \Phi(\mathbf{A}, B), w_{j}\right)_{L^{2}(\Omega)}\langle\Phi(\mathbf{A}, B)\rangle_{\Omega}\right)\right)+O\left(e^{-\lambda \tau}\right)+O\left(\varepsilon^{3}\right) .
\end{gathered}
$$


3. The parameter $\mu$ is arbitrary and the solution does not depend on it. Therefore, we take the condition of tangentiality

$$
\begin{aligned}
& \left.\frac{\partial \mathbf{u}(\tau, \mu)}{\partial \mu}\right|_{\mu=\tau}=0, \\
& \left.\frac{\partial v(\tau, \mu)}{\partial \mu}\right|_{\mu=\tau}=0, \text { for all } \tau .
\end{aligned}
$$

After noticing that terms multiplying $\varepsilon^{2} \tau$ and $\varepsilon^{2}$ in the equation for $u^{k}$ cancel and that in the equation for $v$ from $\mathrm{O}\left(\varepsilon^{2}\right)$ terms only the last two remain, we arrive at the RG equations

$$
\begin{gathered}
\frac{\partial \mathbf{A}(x, \tau)}{\partial \tau}=\varepsilon \mathbf{f}(\mathbf{A}(x, \tau), B(\tau)), \quad \text { for } \tau>0 \\
\frac{d B(\tau)}{d \tau}=\varepsilon\langle\Phi(\mathbf{A}(\cdot, \tau), B(\tau))\rangle_{\Omega}+\varepsilon^{2} \sum_{j=1}^{+\infty} \frac{w_{j}(x)}{\lambda_{j}}\left(\left(\nabla_{u} \Phi(\mathbf{A}, B) \cdot \mathbf{f}(\mathbf{A}, B), w_{j}\right)_{L^{2}(\Omega)}+\right. \\
\left.\left(\partial_{\nu} \Phi(\mathbf{A}, B), w_{j}\right)_{L^{2}(\Omega)}\langle\Phi(\mathbf{A}, B)\rangle_{\Omega}\right), \quad \text { for } \tau>0
\end{gathered}
$$

Returning to the original variable $t=\varepsilon \tau$, we obtain the desired approximation

$$
\begin{gathered}
\frac{\partial \mathbf{A}(x, t)}{\partial t}=\mathbf{f}(\mathbf{A}(x, t), B(t)), \quad \text { for } t>0 \\
\frac{d B(t)}{d t}=\langle\Phi(\mathbf{A}(\cdot, t), B(t))\rangle_{\Omega}+\varepsilon \sum_{j=1}^{+\infty} \frac{w_{j}(x)}{\lambda_{j}}\left(\left(\nabla_{u} \Phi(\mathbf{A}, B) \cdot \mathbf{f}(\mathbf{A}, B), w_{j}\right)_{L^{2}(\Omega)}+\right. \\
\left.\left(\partial_{v} \Phi(\mathbf{A}, B), w_{j}\right)_{L^{2}(\Omega)}\langle\Phi(\mathbf{A}, B)\rangle_{\Omega}\right), \quad \text { for } t>0
\end{gathered}
$$

Note that $x \in \Omega$ is now just a parameter in (34). For $t=O(1)$ the initial time layer effects became negligible and the approximation is expressed by $\mathbf{A}(x, t)$.

The correct behavior for small times is described by the initial time layers, as in the classical literature (see e.g. [19], [27] and [28]).

\section{The shadow limit through energy estimates}

\section{Existence results for the shadow problem}

We start by summarizing properties of the shadow system (4)-(5):

$$
\begin{array}{lll}
\frac{\partial \mathbf{A}}{\partial t}=\mathbf{f}(\mathbf{A}, B, x, t) \quad \text { on } \quad & \Omega \times(0, T) ; & \mathbf{A}(x, 0)=\mathbf{u}^{0}(x) \quad \text { on } \quad \Omega ; \\
\frac{d B}{d t}=\langle\Phi(\mathbf{A}, B, x, t)\rangle_{\Omega} & \text { on } \quad(0, T) ; & B(0)=\left\langle v^{0}\right\rangle_{\Omega},
\end{array}
$$

where $\Omega$ is a bounded open set in $\mathbb{R}^{n}$, with a smooth boundary.

We make the following Assumptions:

A1. $\mathbf{f}$ is a $C^{1}$ function of $x \in \bar{\Omega}$ and a continuous function of $t . \Phi$ is a continuous function in $(x, t) \in \bar{\Omega} \times[0, T]$. 
A2. $\mathbf{f}$ and $\Phi$ are $C^{1}$ functions in $\mathbb{R}^{m+1}$ and locally Lipschitz in $(\mathbf{A}, B) \in \mathbb{R}^{m+1}$. A3. $\mathbf{u}^{0} \in L^{\infty}(\Omega), v^{0} \in H^{1}(\Omega) \cap L^{\infty}(\Omega)$.

Applying Picard iteration to our infinite dimensional setting yields

shlimex Theorem 1 Under Assumptions A1-A3, there is a constant $T_{0}>0$ such that problem (36)-(37) has a unique solution $\{\mathbf{A}, B\} \in C^{1}\left([-T, T], L^{\infty}(\Omega)^{m} \times \mathbb{R}\right)$, for all $T \leq T_{0}$.

Regularity with respect to the space is not restricted to $L^{\infty}(\Omega)$. Let $\mathscr{B}(\Omega)$ be a vector space of all functions defined everywhere on $\Omega$ that are bounded and measurable over $\Omega . \mathscr{B}(\Omega)$ becomes a Banach space when equipped with the norm $\|g\|_{\mathscr{B}(\Omega)}=$ $\sup |g(x)|$.

$x \in \Omega$

bdddef Corollary 1 Let Assumptions Al-A3 hold. Then, if in addition $\mathbf{u}^{0} \in \mathscr{B}(\Omega)^{m}$, there is a constant $T_{0}>0$ such that problem (36)-(37) has a unique solution $\{\mathbf{A}, B\} \in$ $C^{1}\left([-T, T], \mathscr{B}(\Omega)^{m} \times \mathbb{R}\right)$, for all $T \leq T_{0}$. $C(\bar{\Omega})$.

An analogous result, with $\mathscr{B}(\Omega)$ replaced by $C(\bar{\Omega})$, holds if we assume $\mathbf{u}^{0} \in$

Differentiability properties can be shown along the same lines:

H1reg Proposition 1 Let Assumptions Al-A3 hold. Then, if in addition $\mathbf{u}^{0} \in H^{1}(\Omega)^{m}$, there is a constant $T_{0}>0$ such that problem (36)-(37) has a unique solution $\{\mathbf{A}, B\} \in$ $C^{1}\left([-T, T],\left(H^{1}(\Omega)^{m} \cap L^{\infty}(\Omega)^{m}\right) \times \mathbb{R}\right)$, for all $T \leq T_{0}$.

If, in addition to Assumptions A1-A3, we assume

A4. There exist continuous functions $c, k$, defined on $\mathbb{R}$ with values in $\mathbb{R}_{+}$, such that

$$
\|\left.\mathbf{f}(\mathbf{y}, \cdot, t)\right|_{H^{1}(\Omega)^{m}}+\left|\langle\Phi(\mathbf{y}, \cdot, t)\rangle_{\Omega}\right| \leq c(t)+k(t)|\mathbf{y}|_{\mathbb{R}^{m+1}}, \quad \forall \mathbf{y} \in \mathbb{R}^{m+1}
$$

Growth

then every maximal solution to problem (36)-(37) is global.

Auxiliary problems for analysis of the $\varepsilon$-problem.

In the following we introduce two auxiliary problems.

The first problem is linked to the fact that in the shadow limit equation, only the mean of $\Phi$ appears. We have to take care of the replacement of $\Phi$ by its spatial mean and to introduce a correction $w^{\Phi}$ by

$$
\begin{gathered}
-\Delta_{x} w^{\Phi}=\Phi(\mathbf{A}, B, x, t)-\langle\Phi(\mathbf{A}, B, x, t)\rangle_{\Omega} \text { in } \Omega, \\
\frac{\partial w^{\Phi}}{\partial v}=0 \text { on } \partial \Omega, \quad\left\langle w^{\Phi}\right\rangle_{\Omega}=0 .
\end{gathered}
$$

Problem (39)-(40) admits a unique variational solution $w^{\Phi} \in C\left([0, T] ; H^{1}(\Omega)\right)$. Furthermore, $w^{\Phi} \in C\left([0, T] ; W^{2, r}(\Omega)\right)$, for all $r \in[1,+\infty)$.

Next $\partial_{t} w^{\Phi}$ satisfies

$$
\begin{gathered}
-\Delta_{x} \partial_{t} w^{\Phi}=\nabla_{A} \Phi \partial_{t} \mathbf{A}+\partial_{B} \Phi \partial_{t} B+\partial_{t} \Phi-\left\langle\nabla_{A} \Phi \partial_{t} \mathbf{A}\right\rangle_{\Omega}- \\
\left\langle\partial_{B} \Phi \partial_{t} B\right\rangle_{\Omega}-\left\langle\partial_{t} \Phi\right\rangle_{\Omega} \in C\left([0, T] ; L^{\infty}(\Omega)\right) ; \\
\frac{\partial \partial_{t} w^{\Phi}}{\partial v}=0 \text { on } \partial \Omega, \quad\left\langle\partial_{t} w^{\Phi}\right\rangle_{\Omega}=0 .
\end{gathered}
$$


Therefore

$$
\partial_{t} w^{\Phi} \in C\left([0, T] ; W^{2, r}(\Omega)\right), \quad \forall r \in[1,+\infty) .
$$

The second one is linked to the fact that the shadow approximation uses only the space average of the initial value $v^{0}$ of $v$. It creates an initial time layer given by

$$
\begin{gathered}
\left(\frac{\partial}{\partial \tau}-\Delta_{x}\right) \xi^{i}(x, \tau)=0 \quad \text { in } \Omega \times(0,+\infty), \\
\frac{\partial \xi^{i}}{\partial v}=0 \text { on } \partial \Omega \times(0,+\infty), \quad \xi^{i}(x, 0)=v^{0}(x)-\varepsilon w^{\Phi}(x, 0)-\left\langle v^{0}\right\rangle_{\Omega} \text { in } \Omega .
\end{gathered}
$$

Assumption A.3. and the separation of variables for the heat equation yield

$$
\xi^{i}(x, \tau)=\sum_{j=1}^{\infty} e^{-\lambda_{j} \tau}\left(\tilde{v}^{0}, w_{j}\right)_{L^{2}(\Omega)} w_{j}(t) \in L^{2}\left(0, T ; H^{1}(\Omega)\right) \cap C\left([0, T] ; L^{2}(\Omega)\right)
$$

for all finite positive $T$, with $\tilde{v}^{0}=v^{0}-\varepsilon w^{\Phi}(x, 0)$. Furthermore, by a simple comparison principle $\tilde{v}^{0} \in L^{\infty}(\Omega)$ implies $\xi^{i} \in L^{\infty}(\Omega \times(0, T))$. Note that $\tilde{v}^{0} \in H^{1}(\Omega)$ implies $\partial_{t} \xi^{i} \in L^{2}(\Omega \times(0, T))$ and $\xi^{i} \in L^{\infty}\left(0, T ; H^{1}(\Omega)\right)$. In the remainder of this section, we use the initial layer function $\xi^{i, \varepsilon}(x, t)=\xi^{i}\left(x, \frac{t}{\varepsilon}\right)$.

\section{Well-posedness of the $\varepsilon$-problem}

Next, we focus on a short time well-posedness of the $\varepsilon$-problem (1)-(3). Here we consider a more general variant of the problem given by:

$$
\begin{gathered}
\frac{\partial \mathbf{u}_{\varepsilon}}{\partial t}=\mathbf{f}\left(\mathbf{u}_{\varepsilon}, v_{\varepsilon}, x, t\right), \quad \text { in } \quad(0, T) \times \Omega ; \quad \mathbf{u}_{\varepsilon}(0)=\mathbf{u}^{0}(x), x \in \Omega ; \\
\frac{\partial v_{\varepsilon}}{\partial t}-\frac{1}{\varepsilon} \Delta v_{\varepsilon}=\Phi\left(\mathbf{u}_{\varepsilon}, v_{\varepsilon}, x, t\right), \quad \text { in } \quad(0, T) \times \Omega ; \\
v_{\varepsilon}(0)=v^{0}(x), x \in \Omega ; \quad \frac{\partial v_{\varepsilon}}{\partial v}=0 \quad \text { in } \quad(0, T) \times \partial \Omega .
\end{gathered}
$$

Existence of a mild solution for a short time follows from the standard theory, see e.g. the textbook of Henry [13] or ref. [21].

For completeness of the presentation, in the remainder of this section we provide an independent proof of the short time existence and uniqueness of solutions of $\varepsilon$-problem (46)-(48). Using an explicit decomposition of the solution, we link the existence time interval of system (46)-(48), to the existence time interval of the reduced problem (36)-(37). The proposed decomposition provides dependence of the spatial regularity of the solution on the regularity of the initial datum. Moreover, it proves to be useful in the error estimation in Theorem 3 showing that that the time existence interval for variational solutions of problem (46)-(48) is always greater or equal to the existence time interval for problem (36)-(37). 
We start by recalling some classical results on linear parabolic equations from the monograph [17], chapter IV, subsection 9. Let us consider the following problem

$$
\begin{gathered}
\frac{\partial z}{\partial t}-\frac{1}{\varepsilon} \Delta z=F_{\Phi}(x, t), \quad \text { in } \quad(0, T) \times \Omega ; \\
z(0)=0, x \in \Omega ; \quad \frac{\partial z}{\partial v}=0 \quad \text { in } \quad(0, T) \times \partial \Omega .
\end{gathered}
$$

For $F_{\Phi} \in L^{\infty}(\Omega \times(0, T))$, the problem (49)-(50) has smooth solutions. The corresponding functional space for the solutions is

$$
W_{q}^{2,1}\left(Q_{T}\right)=\left\{\phi \in L^{q}\left(Q_{T}\right) \mid \partial_{t}^{r} D_{x}^{s} \phi \in L^{q}\left(Q_{T}\right) \text { for } 2 r+s \leq 2\right\},
$$

with $Q_{T}=\Omega \times(0, T)$ and $1 \leq q<+\infty$.

The solutions are characterized by the following result:

Proposition 2 ([17]) Let $F_{\Phi} \in L^{\infty}(\Omega), q>\max \{3,(n+2) / 2\}$ and $0<\kappa<2-(n+$ $2) / q$. Then the solution to (49)-(50) is an element of $W_{q}^{2,1}\left(Q_{T}\right)$ and

$$
\|z\|_{C^{\kappa, \kappa / 2}\left(\bar{Q}_{T}\right)} \leq C^{\prime}|| z\left\|_{W_{q}^{2,1}\left(Q_{T}\right)} \leq C|| F_{\Phi}\right\|_{L^{q}\left(Q_{T}\right)} .
$$

If $q>n+2$, then $\nabla_{x} z$ is also Hölder continuous in $x$ and $t$.

Now we are ready to prove a local in time existence of unique solutions to system (46)-(48).

prop1 Theorem 2 Let Assumptions A1-A3 hold and let $v^{0} \in H^{1}(\Omega) \cap L^{\infty}(\Omega)$. Then, there exists $T_{0}>0$ such that problem (46)-(48) has a unique solution $\left\{\mathbf{u}_{\varepsilon}, v_{\varepsilon}\right\} \in C^{1}\left([0, T] ; L^{\infty}(\Omega)\right)^{m} \times$ $\left(L^{2}\left(0 ; T ; H^{1}(\Omega)\right) \cap L^{\infty}\left(Q_{T}\right)\right), \partial_{t} v_{\varepsilon} \in L^{2}(\Omega \times(0, T))$.

Proof of Theorem 2.

The proof is based on Schauder's fixed point theorem.

We introduce a convex set

$$
K=\left\{\mathbf{z} \in X=L^{2}\left(0, T ; H^{1}(\Omega)\right) \cap C\left([0, T] ; L^{\infty}(\Omega)\right) \mid\|\mathbf{z}\|_{X} \leq R\right\}
$$

and search for a solution in the form

$$
\begin{aligned}
& \mathbf{u}_{\varepsilon}=\mathbf{A}+\mathbf{U}, \\
& v_{\varepsilon}=B+\varepsilon w^{\Phi}+\xi^{i, \varepsilon}+V .
\end{aligned}
$$

In the decomposition, $\xi^{i, \varepsilon}$ contains the information about the initial condition. $V$ is a smooth function needed for application of the compact embedding in the proof. The decomposition allows proving the result without supposing a high regularity of the initial condition $v^{0}$. Note that for sufficiently small $T$, the functions $\mathbf{A}$ and $B$ are well-defined.

Let $V^{(1)} \in K$. Then we define $\mathbf{U}$ as a solution of the following problem

$\frac{\partial \mathbf{U}}{\partial t}=\mathbf{f}\left(\mathbf{A}+\mathbf{U}, V^{(1)}+B+\varepsilon w^{\Phi}+\xi^{i, \varepsilon}, x, t\right)-\mathbf{f}(\mathbf{A}, B, x, t)$ in $(0, T) ; \mathbf{U}(x, 0)=0$, 
for almost all $x \in \Omega$. Assumptions A1-A3 and boundedness of $w^{\Phi}$ and $\xi^{i, \varepsilon}$ yield that problem (52) has a unique solution $\mathbf{U} \in C^{1}\left([0, T] ; L^{\infty}(\Omega)\right), T \leq T_{0}$, such that $\|\mathbf{U}\|_{C\left(\left[0, T_{0}\right] ; L^{\infty}(\Omega)^{m}\right)} \leq C_{K} T$, where $C_{K}$ depend on $R$, but not on $V^{(1)}$.

Next, we define $V$ as the solution of the equation

$$
\begin{gathered}
\frac{\partial V}{\partial t}-\frac{1}{\varepsilon} \Delta V \\
=\Phi\left(\mathbf{U}+\mathbf{A}, V^{(1)}+B+\varepsilon w^{\Phi}+\xi^{i, \varepsilon}, x, t\right)-\langle\Phi(\mathbf{A}, B, x, t)\rangle_{\Omega}+\Delta w^{\Phi}-\varepsilon \partial_{t} w^{\Phi} \\
=\Phi\left(\mathbf{U}+\mathbf{A}, V^{(1)}+B+\varepsilon w^{\Phi}+\xi^{i, \varepsilon}, x, t\right)-\Phi(\mathbf{A}, B, x, t)-\varepsilon \partial_{t} w^{\Phi} \\
\text { in } \quad(0, T) \times \Omega ; \\
V(x, 0)=0, x \in \Omega ; \quad \frac{\partial V}{\partial v}=0 \quad \text { in } \quad(0, T) \times \partial \Omega .
\end{gathered}
$$

Existence of a unique solution $V \in L^{2}\left(0, T ; H^{1}(\Omega)\right), \partial_{t} V \in L^{2}((0, T) \times \Omega)$ of problem (53)-(54) is straightforward (see e.g. [13]) and the basic energy estimate implies $\|V\|_{L^{\infty}\left(0, T_{0} ; L^{2}(\Omega)\right)}+\|V\|_{L^{2}\left(0, T_{0} ; H^{1}(\Omega)\right)} \leq \frac{R}{2}$, for a sufficiently small $T_{0}$.

Next for sufficiently small $T_{0}$, estimate (51) yields

$$
\|V\|_{C\left([0, T] ; L^{\infty}(\Omega)\right)} \leq \frac{R}{2} .
$$

Now we define the nonlinear mapping $\mathscr{T}: K \rightarrow L^{2}\left(0, T ; H^{1}(\Omega)\right) \cap C\left([0, T] ; L^{\infty}(\Omega)\right)$, $T \leq T_{0}$, by $\mathscr{T}\left(V^{(1)}\right)=V$. The above discussion yields $\mathscr{T}(K) \subseteq K$. Next $\mathscr{T}$ is obviously continuous with respect to the strong topology on $K$. Finally, $\mathscr{T}$ maps $K$ into a ball in $W_{q}^{2,1}\left(Q_{T}\right)$, for any $q<+\infty$. By $(51), W_{q}^{2,1}\left(Q_{T}\right)$ is for $q>\max \{3,(n+2) / 2\}$ compactly imbedded into $C\left(\bar{Q}_{T}\right)$ and, also, into $L^{2}\left(0, T ; H^{1}(\Omega)\right)$. Therefore the range of $\mathscr{T}$ is precompact in $K$ for $T \leq T_{0}$ and, by Schauder's theorem, the problem has at least one fixed point in $K$. Furthermore, from regularity of the ODE solutions we conclude that $\mathbf{U} \in C^{1}\left([0, T] ; L^{\infty}(\Omega)\right)^{m}$.

Now $\mathbf{u}_{\varepsilon}=\mathbf{U}+\mathbf{A} \in C^{1}\left([0, T] ; L^{\infty}(\Omega)\right)^{m}$ and $v_{\varepsilon}=V+B+\varepsilon w^{\Phi}+\xi^{i, \varepsilon} \in$ $L^{2}\left(0 ; T ; H^{1}(\Omega)\right) \cap L^{\infty}\left(Q_{T}\right)$, and $\partial_{t} v_{\varepsilon} \in L^{2}((0, T) \times \Omega)$.

The regularity and Lipschitz property imply uniqueness.

An error estimate for the shadow approximation on finite time intervals

Now we introduce the error functions by

$$
\mathscr{U}_{\varepsilon}=\mathbf{u}_{\varepsilon}-\mathbf{A} \quad \text { and } \quad \mathscr{V}_{\varepsilon}=v_{\varepsilon}-B-\varepsilon w^{\Phi}-\xi^{i, \varepsilon} .
$$

Our goal is to estimate the error functions and to show that they are small in a suitable norm. Such estimates allow to conclude that problems (46)-(48) and (36)-(37) have the same maximal time existence interval. Note that the nonlinearities are Lipschitz functions on any cylinder where a solution exists. 
The function $\mathscr{V}_{\varepsilon}$ is given by

$$
\begin{gathered}
\frac{\partial \mathscr{V}_{\varepsilon}}{\partial t}-\frac{1}{\varepsilon} \Delta \mathscr{V}_{\varepsilon}=\Phi\left(\mathscr{U}_{\varepsilon}+\mathbf{A}, \mathscr{V}_{\varepsilon}+B+\varepsilon w^{\Phi}+\xi^{i, \varepsilon}, x, t\right)-\Phi(\mathbf{A}, B, x, t)-\varepsilon \partial_{t} w^{\Phi} \\
\text { in } \Omega \times(0, T) ; \\
\mathscr{V}_{\varepsilon}(x, 0)=0, x \in \Omega ; \quad \frac{\partial \mathscr{V}_{\varepsilon}}{\partial v}=0 \quad \text { in } \partial \Omega \times(0, T) .
\end{gathered}
$$

We start with an $L^{\infty}$ error estimate.

Our first cut-off function is

$$
\Theta(z)= \begin{cases}-\varepsilon \log (1 / \varepsilon), & \text { for } \quad z<-\varepsilon \log (1 / \varepsilon) \\ z, & \text { for }-\varepsilon \log (1 / \varepsilon) \leq z \leq \varepsilon \log (1 / \varepsilon) \\ \varepsilon \log (1 / \varepsilon), & \text { for } z>\varepsilon \log (1 / \varepsilon)\end{cases}
$$

Next, we write the right hand side in equation (57) as

$$
\begin{gathered}
\Phi\left(\mathscr{U}_{\varepsilon}+\mathbf{A}, \mathscr{V}_{\varepsilon}+B+\varepsilon w^{\Phi}+\xi^{i, \varepsilon}, x, t\right)-\Phi(\mathbf{A}, B, x, t)=\nabla_{A} \Phi(\mathbf{A}, B, x, t) \mathscr{U}_{\varepsilon}+ \\
\partial_{B} \Phi(\mathbf{A}, B, x, t)\left(\mathscr{V}_{\varepsilon}+\varepsilon w^{\Phi}+\xi^{i, \varepsilon}\right)+F\left(\mathscr{U}_{\varepsilon}, \mathscr{V}_{\varepsilon}+\varepsilon w^{\Phi}+\xi^{i, \varepsilon}\right),
\end{gathered}
$$

where $F$ is quadratic in its variables. Following ideas of the center manifold theory (see e.g. [2], we construct a convenient cut-off in $F$. We use the second cut-off function $\rho: \mathbb{R} \rightarrow[0,1]$, being a $C^{\infty}$ function with compact support and satisfying

$$
\rho(\zeta)=\left\{\begin{array}{l}
1, \text { for }|\zeta| \leq 1 \\
0, \text { for }|\zeta| \geq 2
\end{array}\right.
$$

Next we set $\tilde{F}_{\varepsilon}(y, z)=\rho\left(\frac{|z|}{\sqrt{\varepsilon}}\right) F\left(\Theta\left(y_{1}\right), \ldots, \Theta\left(y_{n}\right), z\right)$. It is straightforward to see that $\left|\tilde{F}_{\varepsilon}(y, z)\right|=O(1)|(y, z)|^{2},\left|\frac{d}{d y} \tilde{F}_{\varepsilon}(y)\right|=O(1)|(y, z)|,\left\|\tilde{F}_{\varepsilon}\right\|_{C^{1}}=O(1) \sqrt{\varepsilon},\left\|\tilde{F}_{\varepsilon}\right\|_{C}=O(1) \varepsilon$.

Our cut-off of the higher order terms in (3) is

$$
\begin{gathered}
F_{\varepsilon}(y, z, t)=\rho\left(\frac{|z|}{\sqrt{\varepsilon}}\right) F\left(\Theta\left(y_{1}\right), \ldots, \Theta\left(y_{n}\right), z\right)\left(1-\rho\left(\frac{2 t \lambda_{1}}{-\varepsilon \log \varepsilon}\right)\right)+ \\
\rho(|z|) \rho\left(\frac{2 t \lambda_{1}}{-\varepsilon \log \varepsilon}\right) F\left(\Theta\left(y_{1}\right), \ldots, \Theta\left(y_{n}\right), z\right)
\end{gathered}
$$

A direct calculation gives

Lemma 2 There is a constant $C>0$, independent of $\varepsilon$, such that for all $(y, z, t)$ we have

$$
\left|F_{\varepsilon}(y, t)\right| \leq C \varepsilon+C \mathbb{1}_{\left\{t \leq-\varepsilon \log \varepsilon /\left(2 \lambda_{1}\right)\right\}} .
$$


We search to prove an $L^{\infty}$-bound for $\mathscr{V}_{\varepsilon}$. In order to do it we introduce a problem where the higher order nonlinearities are cut:

$$
\begin{gathered}
\frac{\partial \beta_{\varepsilon}}{\partial t}-\frac{1}{\varepsilon} \Delta \beta_{\varepsilon}=\nabla_{A} \Phi(\mathbf{A}, B, x, t)\left(\Theta\left(\mathscr{U}_{1, \varepsilon}\right), \ldots, \Theta\left(\mathscr{U}_{n, \varepsilon}\right)\right)+\partial_{B} \Phi(\mathbf{A}, B, x, t)\left(\beta_{\varepsilon}+\right. \\
\left.\varepsilon w^{\Phi}+\xi^{i, \varepsilon}\right)-\varepsilon \partial_{t} w^{\Phi}+F_{\varepsilon} \quad \text { in } \quad \Omega \times(0, T) ; \\
\beta_{\varepsilon}(x, 0)=0, x \in \Omega ; \quad \frac{\partial \beta_{\varepsilon}}{\partial v}=0 \quad \text { on } \quad \partial \Omega \times(0, T) .
\end{gathered}
$$

Proposition 3 Let Assumptions Al-A3 hold and let $v^{0} \in H^{1}(\Omega) \cap L^{\infty}(\Omega), \mathbf{u}^{0} \in L^{\infty}(\Omega)^{n}$. Then there exists a constant $C>0$, independent of $\varepsilon$, such that for $\varepsilon \leq \varepsilon_{0}$ we have

$$
\sup _{(x, t) \in \Omega \times(0, T)}\left|\beta_{\varepsilon}(x, t)\right| \leq C \varepsilon \log \left(\frac{1}{\varepsilon}\right) .
$$

Proof We test equation (64) by $\left(\beta_{\varepsilon}-C^{M}(t)\right)_{+}$, where $C^{M}$ is a nonnegative function to be determined. It yields the variational equality

$$
\begin{gathered}
\frac{1}{2} \frac{d}{d t} \int_{\Omega}\left(\beta_{\varepsilon}-C^{M}(t)\right)_{+}^{2} d x+\frac{1}{\varepsilon} \int_{\Omega}\left|\nabla\left(\beta_{\varepsilon}-C^{M}(t)\right)_{+}\right|^{2} d x+ \\
\int_{\Omega}\left(\beta_{\varepsilon}-C^{M}(t)\right)_{+}\left(\frac{d}{d t} C^{M}-\nabla_{A} \Phi(\mathbf{A}, B, x, t)\left(\Theta\left(\mathscr{U}_{1, \varepsilon}\right), \ldots, \Theta\left(\mathscr{U}_{n, \varepsilon}\right)\right)-F_{\varepsilon}+\varepsilon \partial_{t} w^{\Phi}-\right. \\
\left.\partial_{B} \Phi(\mathbf{A}, B, x, t)\left(C^{M}+\varepsilon w^{\Phi}+\xi^{i, \varepsilon}\right)\right) d x=\int_{\Omega} \partial_{B} \Phi(\mathbf{A}, B, x, t)\left(\beta_{\varepsilon}-C^{M}(t)\right)_{+}^{2} d x .
\end{gathered}
$$

Now, if $C^{M}$ is chosen in the way that the third term at the left hand side of (67) is nonnegative, then (65) and Gronwall's inequality would give $\left(\beta_{\varepsilon}-C^{M}(t)\right)_{+}=0$ a.e. on $\Omega \times(0, T)$, i.e. $\beta_{\varepsilon}(x, t) \leq C^{M}(t)$ a.e. on $\Omega \times(0, T)$.

Let us now construct an appropriate barrier function $C^{M}$. We recall that $\nabla_{A} \Phi(\mathbf{A}, B, x, t)$ and $\partial_{B} \Phi(\mathbf{A}, B, x, t)$ are bounded functions. Next, estimate (63) and boundedness of $\partial_{t} w^{\Phi}$ yield that the term in question in nonnegative if

$$
\frac{d}{d t} C^{M}=\mu C^{M}+C_{1}\left(\varepsilon \log \left(\frac{1}{\varepsilon}\right)+\mathbb{1}_{\left\{t \leq-\varepsilon \log \varepsilon /\left(2 \lambda_{1}\right)\right\}}\right) \quad \text { on } \quad(0, T) ; \quad C^{M}(0)=0,
$$

where $\mu=\left\|\partial_{B} \Phi(\mathbf{A}, B, x, t)\right\|_{L^{\infty}(\Omega \times(0, T))}$ and $C_{1}$ is a constant in the estimate for the terms which do not contain $C^{M}$. After integration of Cauchy's problem (68), we find out that $C^{M}(t) \leq C \varepsilon \log \left(\frac{1}{\varepsilon}\right)$ on $(0, T)$. This proves the upper bound in (66).

Proving the lower bound is analogous.

Next, we study the initial value problem for $\mathscr{U}_{\varepsilon}$, defined by (56):

$\frac{\partial \mathscr{U}_{\varepsilon}}{\partial t}=\mathbf{f}\left(\mathbf{A}+\mathscr{U}_{\varepsilon}, \mathscr{V}_{\varepsilon}+B+\varepsilon w^{\Phi}+\xi^{i, \varepsilon}, x, t\right)-\mathbf{f}(\mathbf{A}, B, x, t)$ in $(0, T) ; \mathscr{U}_{\varepsilon}(x, 0)=0$,

for almost all $x \in \Omega$. We write the nonlinearities at the right hand side in the following form:

$$
\begin{gathered}
\mathbf{f}\left(\mathbf{A}+\mathscr{U}_{\varepsilon}, \mathscr{V}_{\varepsilon}+B+\varepsilon w^{\Phi}+\xi^{i, \varepsilon}, x, t\right)-\mathbf{f}(\mathbf{A}, B, x, t)=\nabla_{A} \mathbf{f}(\mathbf{A}, B, x, t) \mathscr{U}_{\varepsilon}+ \\
\partial_{A} \mathbf{f}(\mathbf{A}, B, x, t)\left(\mathscr{V}_{\varepsilon}+\varepsilon w^{\Phi}+\xi^{i, \varepsilon}\right)+\mathbf{G}\left(\mathscr{U}_{\varepsilon}, \mathscr{V}_{\varepsilon}+\varepsilon w^{\Phi}+\xi^{i, \varepsilon}\right),
\end{gathered}
$$


where $\mathbf{G}$ is quadratic in its arguments. As before, we will slightly modify arguments in $\mathbf{G}$ and consider the function $\mathbf{G}_{\varepsilon}$ given by

$$
\mathbf{G}_{\varepsilon}(y, z)=\mathbf{G}\left(\Theta\left(y_{1}\right), \ldots, \Theta\left(y_{n}\right), z\right)
$$

and consider the problem

$$
\begin{gathered}
\frac{\partial \gamma_{\varepsilon}}{\partial t}=\nabla_{A} \mathbf{f}(\mathbf{A}, B, x, t) \gamma_{\varepsilon}+\partial_{A} \mathbf{f}(\mathbf{A}, B, x, t)\left(\beta_{\varepsilon}+\varepsilon w^{\Phi}+\xi^{i, \varepsilon}\right)+ \\
\mathbf{G}_{\varepsilon}\left(\gamma_{\varepsilon}, \beta_{\varepsilon}+\varepsilon w^{\Phi}+\xi^{i, \varepsilon}\right) \text { in }(0, T) ; \quad \gamma_{\varepsilon}(x, 0)=0
\end{gathered}
$$

for almost all $x \in \Omega$. The explicit representation formula for the solutions to the linear nonautonomous systems of ODEs and estimate (66) yield

Lemma 3 The solution $\gamma_{\varepsilon}$ to Cauchy problem (72) satisfies the estimate

$$
\sup _{(x, t) \in \Omega \times(0, T)}\left|\gamma_{\varepsilon}(x, t)\right| \leq C \varepsilon \log \left(\frac{1}{\varepsilon}\right) .
$$

Proposition 4 The functions $\mathscr{U}_{\varepsilon}$ and $\mathscr{V}_{\varepsilon}$, defined by (56), satisfy the $L^{\infty}$ error estimate

$$
\left\|\mathscr{U}_{\varepsilon}\right\|_{L^{\infty}(\Omega \times(0, T))^{m}}+\left\|\mathscr{V}_{\varepsilon}\right\|_{L^{\infty}(\Omega \times(0, T))} \leq C \varepsilon .
$$

Proof Due to estimates (66) and (73), $\left(\gamma_{\varepsilon}, \beta_{\varepsilon}\right)$ satisfies the same equations as $\left(\mathscr{U}_{\varepsilon}, \mathscr{V}_{\varepsilon}\right)$. Therefore, by uniqueness, $\gamma_{\varepsilon}=\mathscr{U}_{\varepsilon}$ and $\beta_{\varepsilon}=\mathscr{V}_{\varepsilon}$. Estimates (66) and (73) imply

$$
\left\|\mathscr{U}_{\varepsilon}\right\|_{L^{\infty}(\Omega \times(0, T))^{m}}+\left\|\mathscr{V}_{\varepsilon}\right\|_{L^{\infty}(\Omega \times(0, T))} \leq C \varepsilon \log \left(\frac{1}{\varepsilon}\right) .
$$

Next we write system (57)-(58), (72) dans la forme

$$
\begin{gathered}
\frac{\partial \mathscr{V}_{\varepsilon}}{\partial t}-\frac{1}{\varepsilon} \Delta \mathscr{V}_{\varepsilon}=\nabla_{A} \Phi\left(\mathbf{A}^{\varepsilon}, B^{\varepsilon}, x, t\right) \mathscr{U}_{\varepsilon}+\partial_{B} \Phi\left(\mathbf{A}^{\varepsilon}, B^{\varepsilon}, x, t\right)\left(\mathscr{V}_{\varepsilon}+\right. \\
\left.\varepsilon w^{\Phi}+\xi^{i, \varepsilon}\right)-\varepsilon \partial_{t} w^{\Phi} \quad \text { in } \quad \Omega \times(0, T) ; \\
\mathscr{V}_{\varepsilon}(x, 0)=0, \quad x \in \Omega ; \quad \frac{\partial \mathscr{U}_{\varepsilon}}{\partial v}=0 \quad \text { on } \quad \partial \Omega \times(0, T) ; \\
\frac{\partial \mathscr{U}_{\varepsilon}}{\partial t}=\nabla_{A} \mathbf{f}\left(\mathbf{A}^{\varepsilon}, B^{\varepsilon}, x, t\right) \mathscr{U}_{\varepsilon}+\partial_{A} \mathbf{f}\left(\mathbf{A}^{\varepsilon}, B^{\varepsilon}, x, t\right)\left(\mathscr{V}_{\varepsilon}+\varepsilon w^{\Phi}+\xi^{i, \varepsilon}\right) \text { in } \Omega \times(0, T) ; \\
\mathscr{U}_{\varepsilon}(x, 0)=0 \quad x \in \Omega,
\end{gathered}
$$

where $\left(\mathbf{A}^{\varepsilon}, B^{\varepsilon}\right)$ are intermediate values. After (67) and (73), we know that the coefficients $\nabla_{A} \Phi, \partial_{B} \Phi, \nabla_{A} \mathbf{f}$ and $\partial_{A} \mathbf{f}$ are uniformly bounded on $\Omega \times(0, T)$, independently of $\varepsilon$. After repeating the calculations from Proposition 3 and Lemma 3, we obtain estimate (74).

It is convenient to decompose it to $\mathscr{V}_{\varepsilon}=\left\langle\mathscr{V}_{\varepsilon}\right\rangle_{\Omega}+H_{\varepsilon},\left\langle H_{\varepsilon}\right\rangle_{\Omega}=0$ and estimate both terms, $\left\langle\mathscr{V}_{\varepsilon}\right\rangle_{\Omega}$ and $H_{\varepsilon}$, separately. yield

Using a constant as a test function in (76) and applying Gronwall's inequality 
Corollary 2 Let $\left(\mathscr{U}_{\varepsilon}, \mathscr{V}_{\varepsilon}\right)$ be given by (69), (57)-(58). Then

$$
\begin{gathered}
\left\|\mathscr{U}_{\varepsilon}\right\|_{L^{\infty}(\Omega \times(0, T))^{m}} \leq C(T) \varepsilon \quad \text { and } \quad\left\|\frac{d}{d t} \mathscr{U}_{\varepsilon}(t)\right\|_{L^{\infty}(\Omega)^{m}} \leq C(T)\left(\varepsilon+e^{-\lambda_{1} t / \varepsilon}\right) ; \\
\left\|\left\langle\mathscr{V}_{\varepsilon}\right\rangle_{\Omega}\right\|_{L^{\infty}(0, T)} \leq C(T) \varepsilon \quad \text { and } \quad\left|\frac{d}{d t}\left\langle\mathscr{V}_{\varepsilon}\right\rangle_{\Omega}(t)\right| \leq C(T)\left(\varepsilon+e^{-\lambda_{1} t / \varepsilon}\right)
\end{gathered}
$$

Next we estimate the perturbation term $H_{\varepsilon}=\mathscr{V}_{\varepsilon}-\left\langle\mathscr{V}_{\varepsilon}\right\rangle_{\Omega}$.

Proposition 5 The perturbation term $H_{\varepsilon}$ satisfies the estimate

$$
\left\|H_{\mathcal{\varepsilon}}\right\|_{L^{\infty}\left(0, T ; L^{2}(\Omega)\right)}^{2}+\frac{1}{\varepsilon}\left\|\nabla H_{\mathcal{\varepsilon}}\right\|_{L^{2}(\Omega \times(0, T))^{n}}^{2} \leq C(T) \varepsilon^{2} .
$$

Proof of Proposition 5. We use $H_{\varepsilon}$ as a test function for equation (57). It yields a standard energy equality

$$
\begin{gathered}
\frac{1}{2} \frac{d}{d t} \int_{\Omega} H_{\varepsilon}^{2} d x+\frac{1}{\varepsilon} \int_{\Omega}\left|\nabla H_{\varepsilon}\right|^{2} d x=\int_{\Omega} \partial_{B} \Phi\left(\mathbf{A}^{\varepsilon}, B^{\varepsilon}, x, t\right) H_{\varepsilon}^{2} d x+ \\
\int_{\Omega}\left(\nabla_{A} \Phi\left(\mathbf{A}^{\varepsilon}, B^{\varepsilon}, x, t\right) \mathscr{U}_{\varepsilon}-\varepsilon \partial_{t} w^{\Phi}+\right. \\
\left.\partial_{B} \Phi\left(\mathbf{A}^{\varepsilon}, B^{\varepsilon}, x, t\right)\left(\left\langle\mathscr{V}_{\varepsilon}\right\rangle_{\Omega}+\varepsilon w^{\Phi}+\xi^{i, \varepsilon}\right)\right) H_{\varepsilon} d x,
\end{gathered}
$$

where $\left(\mathbf{A}^{\varepsilon}, B^{\varepsilon}\right)$ are intermediate values. The nonlinear term at the second line of (83) is a Lipschitz function in the first two arguments and we estimate integrals of products of various components of the approximation by $H_{\varepsilon}$. The leading order terms are

$$
\begin{gathered}
\int_{\Omega} \sqrt{\varepsilon}\left|\xi^{i, \varepsilon}\right| \frac{\left|H_{\varepsilon}\right|}{\sqrt{\varepsilon}} d x \leq \frac{C_{0}}{\varepsilon} \int_{\Omega}\left|H_{\varepsilon}\right|^{2} d x+C_{1} \varepsilon \int_{\Omega}\left|\xi^{i, \varepsilon}\right|^{2} d x \quad \text { and } \\
\int_{\Omega} \sqrt{\varepsilon}\left|\mathscr{U}_{\varepsilon}\right| \frac{\left|H_{\varepsilon}\right|}{\sqrt{\varepsilon}} d x \leq \frac{C_{0}}{\varepsilon} \int_{\Omega}\left|H_{\varepsilon}\right|^{2} d x+C_{1} \varepsilon \int_{\Omega}\left|\mathscr{U}_{\varepsilon}\right|^{2} d x \leq \frac{C_{0}}{\varepsilon} \int_{\Omega}\left|H_{\varepsilon}\right|^{2} d x+C_{1} \varepsilon^{3}
\end{gathered}
$$

Next we take sufficiently small $C_{0}$ and apply Poincaré's inequality $\left\|H_{\mathcal{E}}\right\|_{L^{2}(\Omega)} \leq$ $C_{p}|| \nabla H_{\mathcal{E}} \|_{L^{2}(\Omega)}$ in the energy estimate. It yields

$$
\frac{d}{d t} \int_{\Omega} H_{\varepsilon}^{2} d x+\frac{1}{\varepsilon} \int_{\Omega}\left|\nabla H_{\varepsilon}\right|^{2} d x \leq C \int_{\Omega} H_{\varepsilon}^{2} d x+C \varepsilon^{3}+C \varepsilon \int_{\Omega}\left|\xi^{i, \varepsilon}\right|^{2} d x .
$$

After integrating in time from 0 to $t$ and using the decay in time of $\xi^{i, \varepsilon}$, we obtain the assertion of the Proposition.

coroT Theorem 3 Under Assumptions A1-A3, it holds

$$
\begin{array}{cc}
\left\|\mathbf{u}_{\varepsilon}-\mathbf{A}\right\|_{\left.L^{\infty}(\Omega \times(0, T))\right)} \leq C(T) \varepsilon, & \text { (85) } \\
\left\|\left\langle v_{\varepsilon}\right\rangle_{\Omega}-B\right\|_{L^{\infty}(0, T)} \leq C(T) \varepsilon, & \text { vectErr } \\
\sqrt{\varepsilon}\left\|v_{\varepsilon}-\xi^{i, \varepsilon}-B\right\|_{L^{\infty}(\Omega \times(0, T))}+\left\|\nabla\left(v_{\varepsilon}-\xi^{i, \varepsilon}-\varepsilon w^{\Phi}\right)\right\|_{L^{2}(\Omega \times(0, T))^{n}} \leq C(T) \varepsilon^{3 / 2} & \text { meanErr } \\
\hline \text { perturbErr }
\end{array}
$$


Proof of Theorem 3. The proof is a direct consequence of Corollary 2 and Proposition 5.

Corollary 3 If in addition Assumption A4 holds, then estimates (85)-(87) hold for all $T<+\infty$.

\section{The shadow limit using a local center manifold theorem in Banach spaces}

The weak point of the results obtained in preceding section is that the estimates depend on the length of the time interval $T$. Since our basic tool was Gronwall's inequality, the constants exhibit an exponential dependence on $T$. To obtain estimates for long time intervals, we have to change the strategy.

A good approach is to eliminate the perturbation term $H_{\varepsilon}$ through an estimate independent of $T$. Then $\left\{\mathbf{u}_{\varepsilon},\left\langle\mathscr{V}_{\varepsilon}\right\rangle_{\Omega}\right\}$ satisfies the system

$$
\begin{aligned}
& \frac{d \mathbf{u}_{\varepsilon}}{d t}=\mathbf{f}\left(\mathbf{u}_{\varepsilon}, B_{\varepsilon}+\varepsilon w^{\phi}+\xi^{i, \varepsilon}+H_{\varepsilon}, x, t\right) \text { on } \Omega \times(0, T) ; \mathbf{u}_{\varepsilon}(x, 0)=\mathbf{u}^{0}(x) \text { on } \Omega ; \\
& \frac{d B_{\varepsilon}}{d t}=\left\langle\Phi\left(\mathbf{u}_{\varepsilon}, B_{\varepsilon}+\varepsilon w^{\phi}+\xi^{i, \varepsilon}+H_{\varepsilon}, x, t\right)\right\rangle_{\Omega} \quad \text { on } \quad(0, T) ; \quad B_{\varepsilon}(0)=\left\langle v^{0}\right\rangle_{\Omega} .
\end{aligned}
$$

We note that system (88)-(89) is a nonlocal and nonlinear perturbation of system (36)(37). So its behavior for small $\varepsilon$, at arbitrary times, is linked to the long time behavior of system (36)-(37). We limit our considerations to the case of the autonomous system (36)-(37) in the paragraphs which follow.

The center manifold theorem for system (36)-(37)

We start by proving the center manifold theorem for system (36)-(37). We recall that it is non-local in $x$ and we have to consider (36)-(37) as an ODE in an appropriate Banach space.

Using spectral problem (8) and smoothness of the boundary of the bounded domain $\Omega$, it is easy to prove that there is a smooth orthonormal basis $\left\{\frac{1}{\sqrt{|\Omega|}}, w_{1}, \ldots\right\}$ for $H^{1}(\Omega)$.

The function A can be represented through Fourier series

$$
A_{k}(x, t)=\left\langle A_{k}(\cdot, t)\right\rangle_{\Omega}+\sum_{j=1}^{\infty} A_{k j}(t) w_{j}(x), \quad k=1, \ldots, m,
$$

which converges in $H^{1}(\Omega)$ for every $t \geq 0$.

If we calculate the Fourier coefficients $\left\{A_{k j}\right\}_{k \in\{1, \ldots, m\}, j \in \mathbb{N}}$, then $\mathbf{A}$ is determined.

We study behavior in a neighborhood of an equilibrium point $\left\{\mathbf{A}^{*}, B^{*}\right\}$. For simplicity we assume $\left\{\mathbf{A}^{*}, B^{*}\right\}=0$ and set

$$
\begin{gathered}
\mathbf{f}(\mathbf{A}, B)=\nabla_{A} \mathbf{f}(0) \mathbf{A}+\partial_{B} \mathbf{f}(0) B+\mathbf{g}(\mathbf{A}, B) ; \\
\Phi(\mathbf{A}, B)=\nabla_{A} \Phi(0) \cdot \mathbf{A}+\partial_{B} \Phi(0) B+g_{\Phi}(\mathbf{A}, B) ;
\end{gathered}
$$


where

$$
\begin{aligned}
& \mathbf{g} \in C^{2}, \quad \mathbf{g}(0)=0 \quad \text { and } \quad \nabla_{A, B} \mathbf{g}(0)=0 \\
& g_{\Phi} \in C^{2}, \quad g_{\Phi}(0)=0 \quad \text { and } \quad \nabla_{A, B} g_{\Phi}(0)=0 \text {. }
\end{aligned}
$$

After multiplying equation (36) by $w_{j}$ and integrating over $\Omega$, we obtain the shadow limit ODEs system

$$
\begin{gathered}
\frac{d}{d t} B=\nabla_{A} \Phi(0) \cdot\langle\mathbf{A}\rangle_{\Omega}+\partial_{B} \Phi(0) B+\left\langle g_{\Phi}(\mathbf{A}, B)\right\rangle_{\Omega} ; \\
\frac{d}{d t}\langle\mathbf{A}\rangle_{\Omega}=\nabla_{A} \mathbf{f}(0)\langle\mathbf{A}\rangle_{\Omega}+\partial_{B} \mathbf{f}(0) B+\langle g(\mathbf{A}, B)\rangle_{\Omega} ; \\
\langle\mathbf{A}\rangle_{\Omega}(0)=\left\langle\mathbf{u}^{0}\right\rangle_{\Omega} ; \quad B(0)=\left\langle v^{0}\right\rangle_{\Omega} . \\
\frac{d}{d t} A_{k j}(t)=\sum_{r=1}^{m}\left(\nabla_{A} \mathbf{f}(0)\right)_{k r} A_{r j}+\left(g_{k}(\mathbf{A}, B), w_{j}\right)_{L^{2}(\Omega)}, \quad k=1, \ldots, m, j \geq 1 ; \\
A_{k j}(0)=\left(u_{k}^{0}, w_{j}\right)_{L^{2}(\Omega)} ;
\end{gathered}
$$

The unknowns are $B$ and the Fourier coefficients from (90).

Next we introduce the operator $L$, which denotes the linearization of our shadow limit ODE system:

- $L$ is defined on the Hilbert space $W=\mathbb{R}^{m+1} \oplus \ell^{2}(\mathbb{N})^{m}$, with $\ell^{2}(\mathbb{N})=\left\{\mathbf{z}=\left(z_{1}, \ldots,\right) \mid\right.$ $\left.\sum_{j=1}^{\infty} z_{j}^{2}<+\infty\right\}$, as a block-diagonal operator

$$
L \varphi=\oplus_{k=0}^{+\infty}(L \varphi)^{k}, \quad \varphi=\left\{b_{0}, \mathbf{a}^{0}, \mathbf{a}^{1}, \ldots\right\} .
$$

$\mathbf{a}^{k}=\left(a_{1 k}, \ldots, a_{m k}\right)$ contains the components of the $k$ th Fourier coefficient and the blocks $(L \varphi)^{j}$ are given by

$$
\begin{gathered}
(L \varphi)^{0}=\Lambda_{0}\left[\begin{array}{l}
b_{0} \\
\mathbf{a}^{0}
\end{array}\right]=\left[\begin{array}{cc}
\partial_{B} \Phi(0) & \nabla_{A} \Phi(0) \\
\partial_{B} \mathbf{f}(0) & \nabla_{A} \mathbf{f}(0)
\end{array}\right]\left[\begin{array}{l}
b_{0} \\
\mathbf{a}^{0}
\end{array}\right] \\
(L \varphi)^{j}=\nabla_{A} \mathbf{f}(0) \mathbf{a}^{j}, \quad j=1,2, \ldots
\end{gathered}
$$

- First block corresponds to the restriction of $L$ to $\mathbb{R}^{m+1}$. It corresponds to the linearized ODEs for $B$ and $<\mathbf{A}>_{\Omega}$. Obviously, $L$ maps $\mathbb{R}^{m+1}$ into itself. Next blocks are built from $m$ times $m$ matrices, corresponding to the Fourier coefficients $\mathbf{A}_{k}$. Invariance is again obvious.

- Hence, $L$ is a bounded linear operator, defined on $W$ with values in the same space.

Let us study the spectrum of $L$ :

If $L \alpha=0, \alpha \neq 0$, then either $\nabla_{A} \mathbf{f}(0)$ has a zero eigenvalue or $\Lambda_{0}$ has it or both. Due to the block structure, $L$ is surjective if and only if it is injective. Consequently, its spectrum contains only eigenvalues and their number is smaller or equal to $2 m+1$. We write the spectrum $\sigma$ as $\sigma=\sigma_{+} \cup \sigma_{c} \cup \sigma_{-}$, where

- $\sigma_{+}=\{\lambda \in \sigma \mid \Re \lambda>0\}$ (the unstable spectrum).

- $\sigma_{c}=\{\lambda \in \sigma \mid \Re \lambda=0\}$ (the central spectrum). 
- $\sigma_{+}=\{\lambda \in \sigma \mid \Re \lambda<0\}$ (the stable spectrum).

In order to construct the center manifold description for problem (36)-(37) we use the theory from the book of Haragus and Iooss [12], chapter 2. In addition to the above established properties of the operator $L$ and the functional space $W$, one has to check the following hypothesis

Spectral decomposition hypothesis ([12], page 31) The set $\sigma_{c}$ consists of a finite number of eigenvalues with finite algebraic multiplicities.

Note that if 0 is an eigenvalue for $\nabla_{A} \mathbf{f}(0)$, then the corresponding eigenspace is infinite dimensional. Hence we assume

A5. All eigenvalues of $\nabla_{A} \mathbf{f}(0)$ are with non-zero real part. $\sigma_{c}$ is linked to $\Lambda_{0}$ and it is not empty.

Next let $P_{c} \in \mathscr{L}(W)$ be the spectral projector corresponding to $\sigma_{c}$. Then

$$
P_{c}^{2}=P_{c}, \quad P_{c} L u=L P_{c} u, \quad \forall u \in W \quad \text { and } \quad \operatorname{Im} P_{c} \text { is finite dimensional. }
$$

Let $P_{h}=I-P_{c}$. Then

$$
P_{h}^{2}=P_{h}, \quad P_{h} L u=L P_{h} u, \quad \forall u \in W \quad \text { and } \quad P_{h} \in \mathscr{L}(W) .
$$

Let $\mathscr{E}_{0}=\operatorname{Im} P_{c}=\operatorname{Ker} P_{h} \subset W, W_{h}=\operatorname{Im} P_{h}=\operatorname{Ker} P_{c} \subset W$. Then, it holds

$$
W=\mathscr{E}_{0} \oplus W_{h} .
$$

Obviously, $\operatorname{dim} \mathscr{E}_{0} \leq m+1$ and it is linked to the eigenvalues of $\Lambda_{0}$ with $\Re \lambda=0$. Assumption A5 yields existence of $\gamma>0$ such that

$$
\inf _{\lambda \in \sigma_{+}} \Re \lambda>\gamma \text { and } \sup _{\lambda \in \sigma_{-}} \Re \lambda<-\gamma .
$$

Let $\eta \in[0, \gamma]$ and

$$
\mathscr{C}_{\eta}(\mathbb{R}, W)=\left\{u \in C(\mathbb{R}, W) \mid \quad\|u\|_{\mathscr{C}_{\eta}}=\sup _{t \in \mathbb{R}}\left(e^{-\eta t}\|u(t)\|_{W}\right)<+\infty\right\} .
$$

$\mathscr{C}_{\eta}(\mathbb{R}, W)$ is a Banach space.

We search to solve the evolution problem

$$
\frac{d u_{h}}{d t}=L_{h} u_{h}+f(t)
$$

in $\mathscr{C}_{\eta}(\mathbb{R}, W)$ and to prove that it defines a linear map $K_{h}, K_{h} f=u_{h}$, which is continuous from $\mathscr{C}_{\eta}(\mathbb{R}, W)$ to itself. $L_{h}$ is the restriction of $L$ to $W_{h}=P_{h} W$.

First we remark that the initial value is determined by the exponential growth and $\mathbf{A}_{j}, j \geq 1$, are given by

$$
\begin{gathered}
\mathbf{A}_{j}(t)=-\int_{t}^{+\infty} e^{\nabla_{A} \mathbf{f}(0)(t-s)} P_{+} f_{j}(s) d s+\int_{-\infty}^{t} e^{\nabla_{A} \mathbf{f}(0)(t-s)} P_{-} f_{j}(s) d s, \\
\mathbf{A}_{j}=\left(A_{1 j}, \ldots, A_{m j}\right), j \geq 1 .
\end{gathered}
$$


For the remaining part we have

$$
\frac{d}{d t} P_{h}\left[\begin{array}{c}
\langle\mathbf{A}\rangle_{\Omega} \\
B
\end{array}\right]=\left[\begin{array}{cc}
\nabla_{A} \mathbf{f}(0) & \partial_{B} \mathbf{f}(0) \\
\nabla_{A} \Phi(0) & \partial_{B} \Phi(0)
\end{array}\right] P_{h}\left[\begin{array}{c}
\langle\mathbf{A}\rangle_{\Omega} \\
B
\end{array}\right]+\left[\begin{array}{c}
f_{A} \\
f_{B}
\end{array}\right]
$$

Since all problems are finite dimensional, we have a unique solution $u_{h} \in \mathscr{C}_{\eta}\left(\mathbb{R}, W_{h}\right)$ and $K_{h}$ is defined by setting $u_{h}=K_{h} f$. Continuity of $K_{h}$ is obvious.

Consequently, we have checked all assumptions of Theorem 2.9 from book [12], i.e. the continuity of the operator $L$ and choice of the functional space $W$, the spectral decomposition, following from Assumption A5, and the solvability of problem (98). We conclude

CMTHI1 Proposition 6 Let Assumptions A1-A3, A5 hold. Then there exists a map $\Psi \in C^{1}\left(\mathscr{E}_{0}, W_{h}\right)$ with

$$
\Psi(0)=0, \quad \nabla \Psi(0)=0,
$$

and a neighborhood $\mathscr{N}$ of 0 in $W=\mathbb{R}^{m+1} \oplus \ell^{2}(\mathbb{N})^{m}$ such that the local center manifold

$$
\mathscr{M}_{0}=\left\{u_{c}+\Psi\left(u_{c}\right) \mid u_{c} \in \mathscr{E}_{0}\right\} \subset W
$$

has the following properties:

(i) $\mathscr{M}_{0}$ is locally invariant, i.e. if $u=(B, \mathbf{A})=\left(b_{0}, \mathbf{a}^{0}, \mathbf{a}^{1}, \ldots\right)$ is a solution for (93)(97) (and consequently for (36)-(37)) satisfying $u(0) \in \mathscr{M}_{0} \cap \mathscr{N}$ and $u(t) \in \mathscr{N}$ for all $t \in[0, T]$, then $u(t) \in \mathscr{M}_{0}$ for all $t \in[0, T]$.

(ii) $\mathscr{M}_{0}$ contains the set of bounded solutions of (36)-(37) staying in $\mathscr{N}$ for all $t \in \mathbb{R}$, i.e. if $u$ is a solution of (36)-(37) satisfying $u(t) \in \mathscr{N}$ for all $t \in \mathbb{R}$, then $u(0) \in \mathscr{M}_{0}$.

effDDE1 Corollary 4 Let the assumptions of Proposition 6 hold. Then every solution $u=$ $(B, \mathbf{A})$ for (36)-(37) which belongs to $\mathscr{M}_{0}$ for all $t \in(0, T) \subset \mathbb{R}$ is of the form $u=$ $u_{c}+\Psi\left(u_{c}\right)$ and $u_{c}$ satisfies

$$
\frac{d}{d t} u_{c}=L_{c} u_{c}+P_{c}\left[\begin{array}{c}
\mathbf{g} \\
g_{\Phi}
\end{array}\right]
$$

where $L_{c}$ is the restriction of $L$ on $\mathscr{E}_{0}$. Furthermore, the reduction function satisfies equation

$$
\frac{d}{d t} \Psi\left(u_{c}\right)=L_{h} \Psi\left(u_{c}\right)+P_{h}\left[\begin{array}{c}
\mathbf{g} \\
g_{\Phi}
\end{array}\right]
$$

The center manifold construction for system (1)-(3)

Now we present the center manifold construction for system (1)-(3). In order to simplify the notation, we denote $\mathbf{u}_{\varepsilon}$ by $\mathbf{A}$.

Next, the unknown function $B$ is replaced by $v_{\varepsilon}$, which we expand as

$$
v_{\varepsilon}=B_{0}+\sum_{j=1}^{\infty} B_{j}(t) w_{j}(x) .
$$


After multiplying equation (1) by $w_{j}$ and integrating over $\Omega$, we obtain

$$
\begin{gathered}
\frac{d}{d t} A_{k j}(t)=\sum_{r=1}^{m}\left(\nabla_{A} \mathbf{f}(0)\right)_{k r} A_{r j}+\left(\partial_{B} \mathbf{f}(0)\right)_{j} B_{j}+\left(g_{k}(\mathbf{A}, B), w_{j}\right)_{L^{2}(\Omega)}, \\
k=1, \ldots, m, j \geq 1 ; \\
A_{k j}(0)=\left(u_{k}^{0}, w_{j}\right)_{L^{2}(\Omega)} ; \\
\frac{d}{d t}\langle\mathbf{A}\rangle_{\Omega}=\nabla_{A} \mathbf{f}(0)\langle\mathbf{A}\rangle_{\Omega}+\partial_{B} \mathbf{f}(0) B_{0}+\langle g(\mathbf{A}, B)\rangle_{\Omega} ; \quad\langle\mathbf{A}\rangle_{\Omega}(0)=\left\langle\mathbf{u}^{0}\right\rangle_{\Omega} .
\end{gathered}
$$

Next we multiply equation (2) by $w_{j}$ and integrate over $\Omega$. It yields

$$
\begin{gathered}
\frac{d}{d t} B_{0}=\nabla_{A} \Phi(0) \cdot\langle\mathbf{A}\rangle_{\Omega}+\partial_{B} \Phi(0) B_{0}+\left\langle g_{\Phi}(\mathbf{A}, B)\right\rangle_{\Omega} ; \quad B_{0}(0)=\left\langle v^{0}\right\rangle_{\Omega} \\
\frac{d}{d t} B_{j}(t)=\left(\partial_{B} \Phi(0)-\frac{\lambda_{j}}{\varepsilon}\right) B_{j}(t)+\nabla_{A} \Phi(0) \cdot \mathbf{A}_{j}+\left(g_{\Phi}(\mathbf{A}, B), w_{j}\right)_{L^{2}(\Omega)} \\
B_{j}(0)=\left(v^{0}, w_{j}\right)_{L^{2}(\Omega)}, j \geq 1
\end{gathered}
$$

The new operator $L^{1}$ is is defined on $W^{1}=\mathbb{R}^{m+1} \oplus \ell^{2}(\mathbb{N})^{m+1}$, as a block-diagonal operator. For $\varphi=\left\{b_{0}, \mathbf{a}^{0}, \mathbf{a}^{1}, b_{1}, \mathbf{a}^{2}, b_{2}, \ldots\right\}$, with $\mathbf{a}^{k}=\left(a_{1 k}, \ldots, a_{m k}\right)$, the block $\left(L^{1} \varphi\right)^{k}$ is defined as follows:

First block corresponds to the restriction of $L^{1}$ to $\mathbb{R}^{m+1}$ and it reads as before:

$$
\left(L^{1} \varphi\right)^{0}=\left.L^{1}\right|_{\mathbb{R}^{m+1}} \varphi=\Lambda_{0}\left[\begin{array}{l}
b_{0} \\
\mathbf{a}^{0}
\end{array}\right] .
$$

Obviously, $L^{1}$ maps $\mathbb{R}^{m+1}$ into itself. Next blocks are slightly different and built from $m+1$ times $m+1$ matrices, corresponding to the action of $L^{1}$ on $\left\{\mathbf{a}^{k}, b^{k}\right\}$. They read

$$
\Lambda_{1}=\left[\begin{array}{cc}
\nabla_{A} \mathbf{f}(0) & \partial_{B} \mathbf{f}(0) \\
\nabla_{A} \Phi(0) & \partial_{B} \Phi(0)-\frac{\lambda_{j}}{\varepsilon}
\end{array}\right] .
$$

Invariance is again obvious. Hence, $L^{1}$ is a bounded linear operator, defined on $W$ with values in the same space.

Since

$$
\operatorname{det}\left(\Lambda_{1}-\lambda I_{m+1}\right)=\operatorname{det}\left(\Lambda_{0}-\lambda I_{m+1}\right)-\frac{\lambda_{1}}{\varepsilon} \operatorname{det}\left(\nabla_{A} \mathbf{f}(0)-\lambda I_{m}\right),
$$

the classical perturbation theory for the eigenvalues yields that there is $q>0$ such that the first $m$ eigenvalues of matrix $\Lambda_{1}$ correspond to an $O\left(\varepsilon^{1 / q}\right)$ perturbation of the eigenvalues of $\nabla_{A} \mathbf{f}(0)$. Using assumption A5, for $\varepsilon \leq \varepsilon_{0}$, we obtain again that the real parts of these eigenvalues are different from zero. Finally, the last eigenvalue is $-\lambda_{j} / \varepsilon+O(1)$ and belongs to $\sigma_{-}$. Again, problem (98) has a unique solution and Theorem 2.9, page 34, [12] holds true. Hence we have an analogue of Proposition 6 and Corollary 4. We note that in both case we have the same space $\mathscr{E}_{0}$. The new equation (102), for $u_{c}^{1}$, differs only in the nonlinear part. Equation (103) now reads

$$
\frac{d}{d t} \Psi\left(u_{c}^{1}\right)=L_{h} \Psi\left(u_{c}^{1}\right)+P_{h}\left[\begin{array}{c}
\mathbf{g} \\
g_{\Phi}
\end{array}\right] .
$$


Theorem 4 Let assumptions of Proposition 6 hold. Then, every solution of problem (1)-(3), which belongs to $\mathscr{M}_{0}$ for all $t \in(0, T) \subset \mathbb{R}$, is of the form $u^{1}=u_{c}^{1}+\Psi\left(u_{c}^{1}\right)$. Functions $B_{j}$ tend exponentially to a corresponding solution of system (1)-(3) on $\mathscr{M}_{0}$ and $\left\|B_{j}\right\|_{L^{1}\left(\mathbb{R}_{+}\right)} \leq C \varepsilon$. Moreover, the distance between the bounded solutions of problem (1)-(3) and its shadow approximation (36)-(37) with the same initial conditions is smaller than $C \varepsilon$ in $L^{\infty}\left(\mathbb{R}_{+}\right)$.

Proof of Theorem 4. The result is a consequence of the term $-\lambda_{j} B_{j} / \varepsilon$ in equation (108).

Acknowledgements Andro Mikelić would like to express his thanks to The Heidelberg Graduates School HGS MathComp, IWR, Universität Heidelberg, for partially supporting his post-Romberg professorship research visits in 2014-2015. AMC acknowledges the support of the Emmy Noether Programme of the German Research Council (DFG).

\section{References}

Bobrowski

Harioos

Henry

Hock

Keener78

Klika

Ladyparab

Epstein

1. A. Bobrowski, Singular perturbations involving fast diffusion, Journal of Mathematical Analysis and Applications 427, 1004-1026 (2015).

2. J. Carr, Applications of Centre Manifold Theory, Springer- Verlag, New York, 1981.

3. L. Y. Chen, N. Goldenfeld, Y. Oono, Renormalization group theory for global asymptotic analysis, Phys. Rev. Lett. 73 , 1311-1315 (1994).

4. L. Y. Chen, N .Goldenfeld, Y. Oono, Renormalization group and singular perturbations: Multiple scales, boundary layers, and reductive perturbation theory, Phys. Rev. E 54, 376-394 (1996).

5. H. Chiba, $C^{1}$ approximation of vector fields based on the renormalization group method, SIAM J. Appl. Dyn. Syst., 7 , 895-932 (2008).

6. H. Chiba, Extension and unification of singular perturbation methods for ODEs based on the renormalization group method, SIAM J. Appl. Dyn. Syst., 8, 1066-1115 (2009).

7. H.Chiba, Reduction of weakly nonlinear parabolic partial differential equations. J. Math. Phys. 54, 101501 (2013).

8. P. Gray and S. K. Scott, Autocatalytic reactions in the isothermal continuous stirred tank reactor: isolas and other forms of multistability. Chem. Eng. Sci. 38 (1983), 29-43.

9. R. E. Lee DeVille, A. Harkin, M. Holzer, K. Josić, T. Kaper, Analysis of a renormalization group method and normal form theory for perturbed ordinary differential equations, Physica D, 237,1029 1052 (2008).

10. A. Gierer, H. Meinhardt, A theory of biological pattern formation, Kybernetik, Vol. 12 (1972), 30-39.

11. J. K. Hale, K. Sakamoto, Shadow systems and attractors in reaction-diffusion equations, Applicable Analysis, 32 , 287-303 (1989).

12. M. Haragus, G. Iooss, Local Bifurcations, Center Manifolds, and Normal Forms in InfiniteDimensional Dynamical Systems, Springer Universitext 2011.

13. D. Henry, Geometric Theory of semilinear Parabolic Equations. Lecture Notes in Math. 840, SpringerVerlag, 1981.

14. S. Hock, Y. Ng, J. Hasenauer, D. Wittmann, D. Lutter, D. Trumbach, W. Wurst, N. Prakash, and F.J.. Theis Sharpening of expression domains induced by transcription and microRNA regulation within a spatio-temporal model of mid-hindbrain boundary formation. BMC Syst Biol 7 (2013) 48.

15. J.P. Keener, Activators and Inhibitors in Pattern Formation, Studies in Applied Mathematics, Vol. 59 , 1-23 (1978).

16. V. Klika, R.E.. Baker, D. Headon, E.A. Gaffney, The influence of receptor-mediated interactions on reaction-diffusion mechanisms of cellular self-organization, Bull. Math. Biol. 74 (2012), 935-957.

17. O. A. Ladyzenskaja, V. A. Solonnikov, N. N. Ural'ceva, Linear and Quasi-linear Equations of Parabolic Type, (Translations of Mathematical Monographs Reprint Vol. 23) American Mathematical Society, Providence, 1968.

18. I. Lengyel, I. R. Epstein, A chemical approach to designing Turing patterns in reaction-diffusion systems, Proc. Natl. Acad. Sci. USA 89 (1992), 3977-3979. 
OM:91 19. R. E. O’Malley, Jr., Singular perturbation methods for ordinary differential equations, Applied mathematical sciences 89, Springer, New York, Berlin, Heidelberg, 1991

dynspike 20. A. Marciniak-Czochra, S. Härting, G. Karch, K. Suzuki, Dynamical spike solutions in a nonlocal model of pattern formation, arXiv:1307.6236 [math.AP] (2013).

MKS12 21. A. Marciniak-Czochra, G. Karch, K. Suzuki Unstable patterns in reaction-diffusion model of early carcinogenesis, J. Math. Pures Appl. 99 (2013), 509-543.

MCK07 22. A. Marciniak-Czochra, M. Kimmel, Modelling of early lung cancer progression: influence of growth factor production and cooperation between partially transformed cells, Math. Models Methods Appl. Sci. 17 (2007), suppl., 1693-1719.

MCK08 23. A. Marciniak-Czochra, M. Kimmel, Reaction-diffusion model of early carcinogenesis: the effects of influx of mutated cells, Math. Model. Nat. Phenom. 3 (2008), 90-114.

MurrayII 24. J.D. Murray, Mathematical biology. II. Spatial models and biomedical applications. Third edition., Interdisciplinary Applied Mathematics 18, Springer-Verlag, New York, 2003.

MoisZiane 25. I. Moise, M. Ziane, Renormalization Group Method. Applications to Partial Differential Equations, Journal of Dynamics and Differential Equations, 13 , 275-321 (2001).

TemWir 26. R. Temam, D. Wirosoetisno, On the solutions of the renormalized equations at all orders, Adv. Diff. Eqn., 8 , 1005-1024 (2003).

Tikh:85 27. A.N. Tikhonov, A.B. Vasil'eva, A.G. Sveshnikov, Differential equations, (Translation of: Differentsial'nye uravneniiya), Springer, Berlin, 1985.

Vasileva:93 28. A. B. Vasil'eva, V. F. Butuzov, L. V. Kalachev, The Boundary Function Method for Singular Perturbed Problems, SIAM, Philadelphia, 1993.

Ziane1 29. M. Ziane, On a certain renormalization group method, J. Math. Phys., 41, 3290-3299 (2000). 